\title{
Trajectory Design Leveraging Low-thrust, Multi-Body \\ Equilibria and Their Manifolds
}

Andrew D. Cox | Kathleen C. Howell | David C. Folta

August 21, 2018

AAS/AIAA Astrodynamics Specialist Conference

Snowbird, UT 


\section{Motivation}

- Low-thrust trajectory design: require position, velocity, \& control histories for initial guess

- Many current methods leverage optimization $\rightarrow$ point solution

- Chaotic multi-body regimes; initial guess may strongly bias result

- Ballistic designs benefit from available dynamical structures but supply no initial control history

Seek a more general understanding of low-thrust + multi-body dynamics 


\section{Roadmap}

CR3BP

CR3BP-LT

Design

Low-Thrust

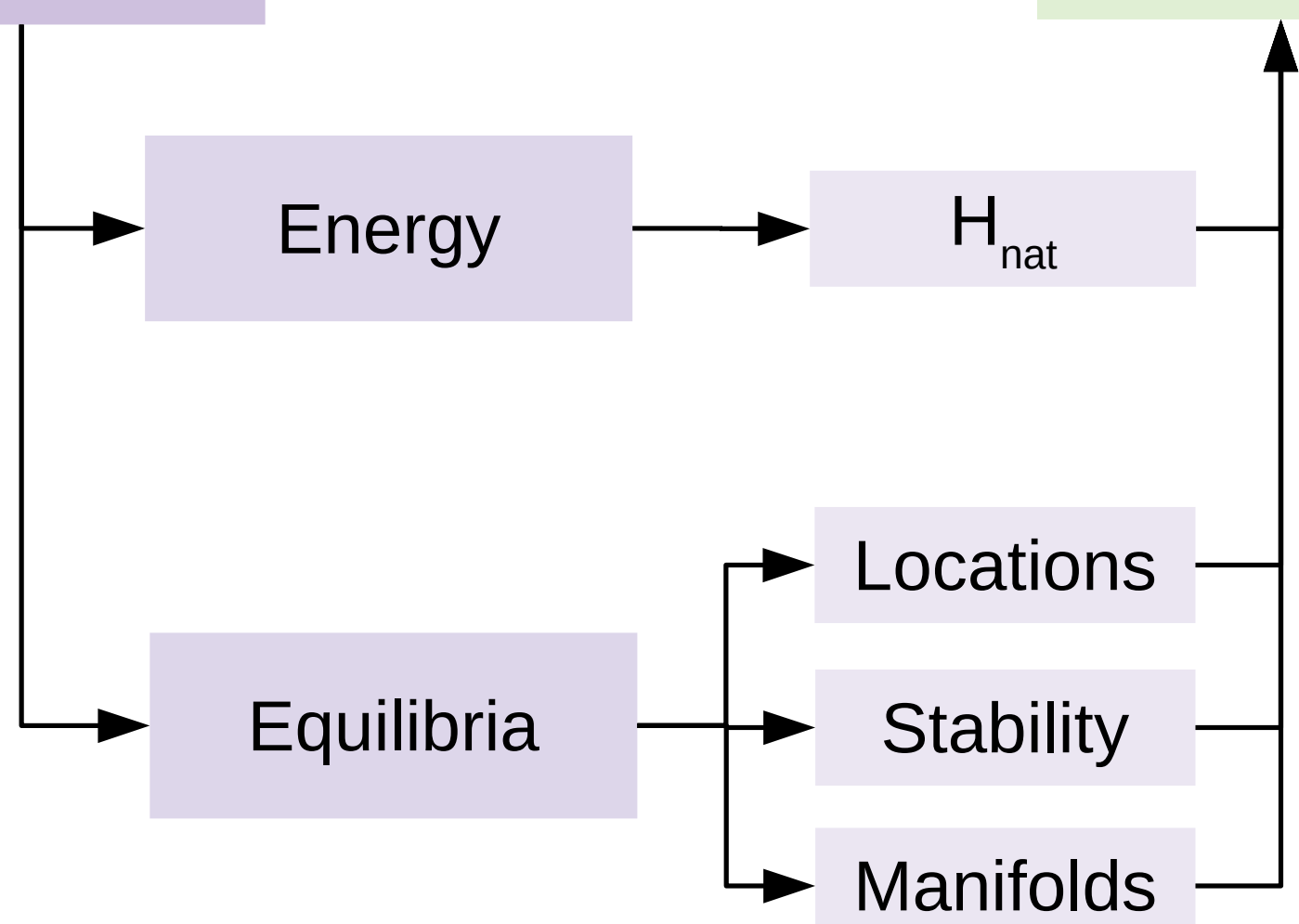




\title{
Roadmap
}

CR3BP

\section{CR3BP-LT}

\author{
Low-Thrust
}




\section{Combined Model}

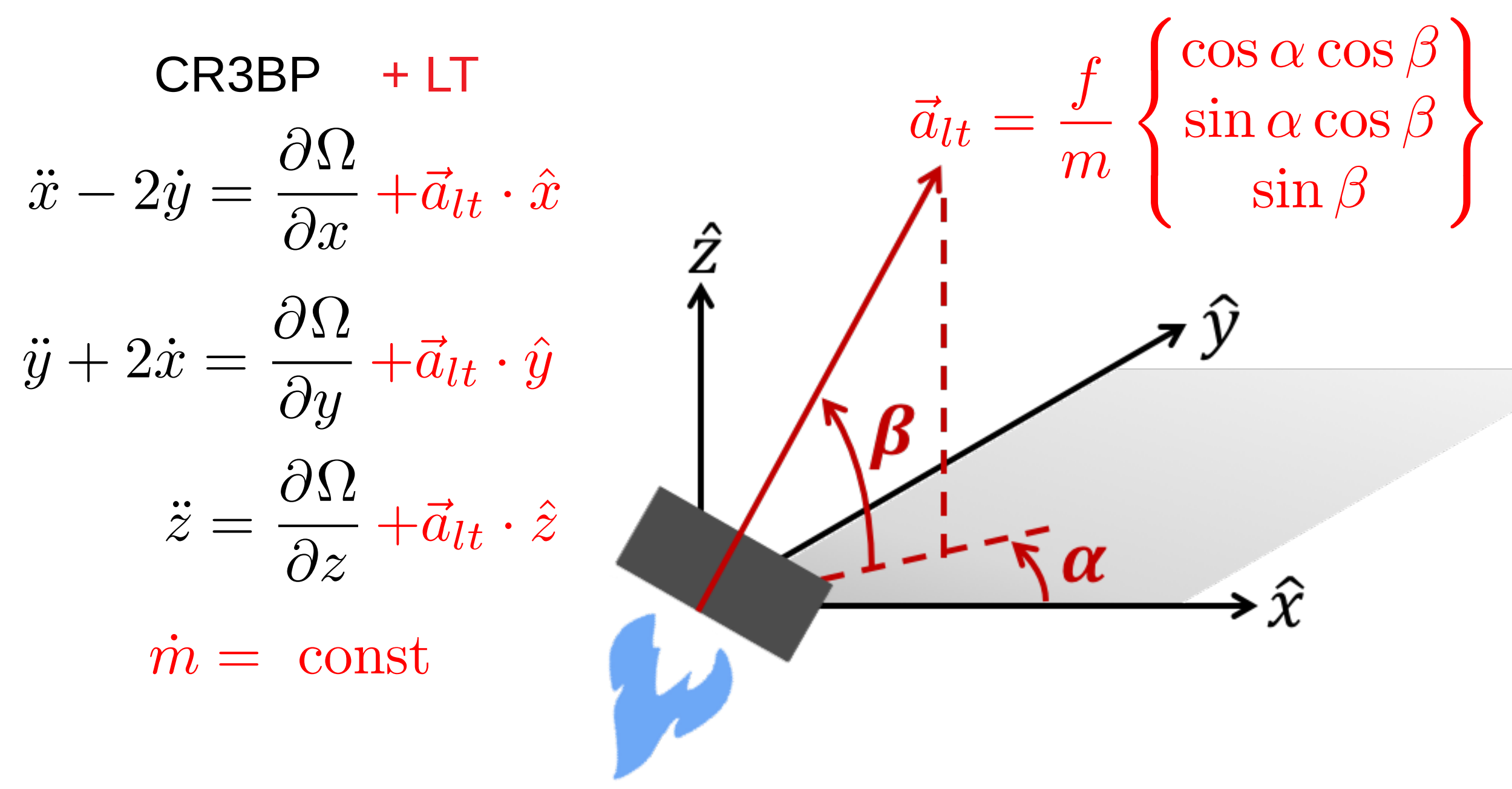




\section{Simplifications}

- $a_{l t}=\frac{f}{m}=$ constant (reasonable in Earth-Moon CR3BP-LT)

- Planar motion: $z=\dot{z}=0, \beta=0$

$$
\begin{aligned}
& \ddot{x}-2 \dot{y}=\frac{\partial \Omega}{\partial x}+a_{l t} \cos \alpha \\
& \ddot{y}+2 \dot{x}=\frac{\partial \Omega}{\partial y}+a_{l t} \sin \alpha
\end{aligned}
$$

Conservative Autonomous Hamiltonian 


\section{Roadmap}

CR3BP

CR3BP-LT

Low-Thrust

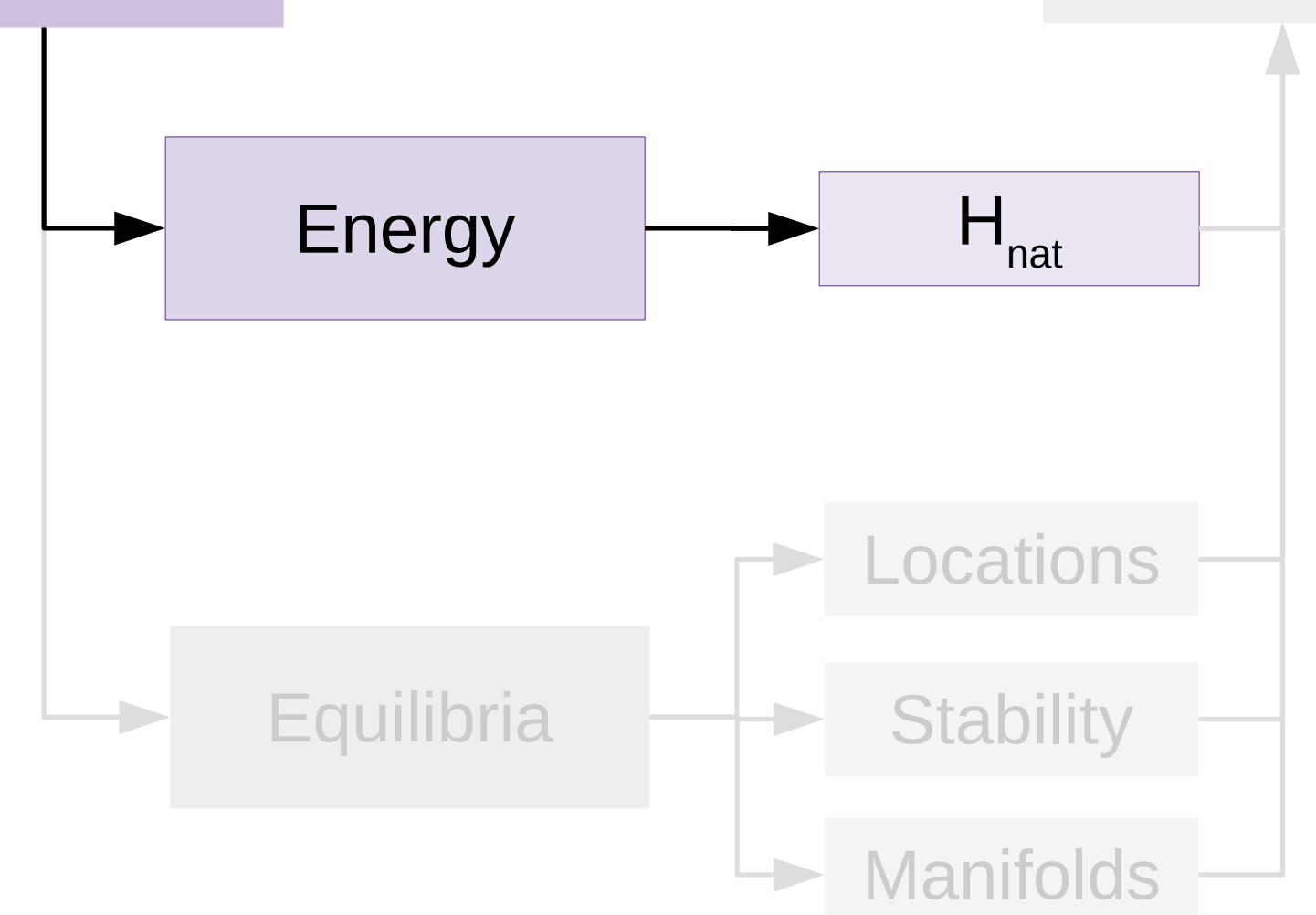




\section{Energy-Like Integral}

Hamiltonian: $\quad H=\sum_{i=1}^{3}\left(p_{i} \dot{g}_{i}\right)-L(\vec{g}, \dot{\vec{g}}, \tau)$

CR3BP (natural) system:

$$
\begin{gathered}
H_{\text {nat }}=\frac{1}{2}\left(\dot{x}^{2}+\dot{y}^{2}+\dot{z}^{2}\right)-\frac{1}{2}\left(x^{2}+y^{2}\right)-\frac{1-\mu}{r_{13}}-\frac{\mu}{r_{23}}=-\frac{1}{2} C \\
\text { Constant on ballistic arcs }
\end{gathered}
$$

Varies on low-thrust arcs independent of path

$$
H_{n a t}\left(t_{f}\right)-H_{n a t}\left(t_{0}\right)=\left[\vec{r}\left(t_{f}\right)-\vec{r}\left(t_{0}\right)\right] \cdot \vec{a}_{l t}
$$




\section{Energy Plane}

Control Point: $\vec{\rho}=\left\{\begin{array}{lll}x & y & H_{n a t}\end{array}\right\}$

Every low-thrust arc (with single $\mathrm{a}_{\mathrm{lt}} \& \alpha$ ) is confined to an energy plane
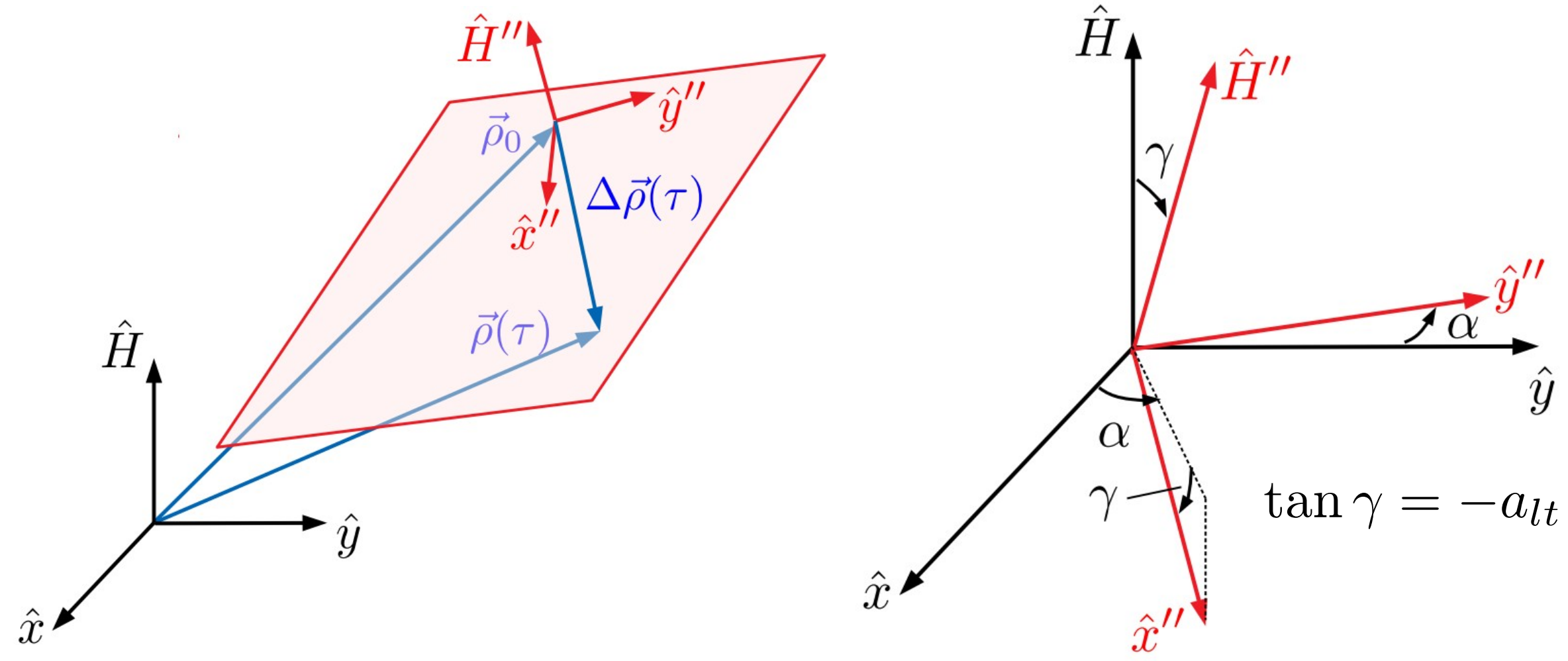


\section{Energy Plane Example}
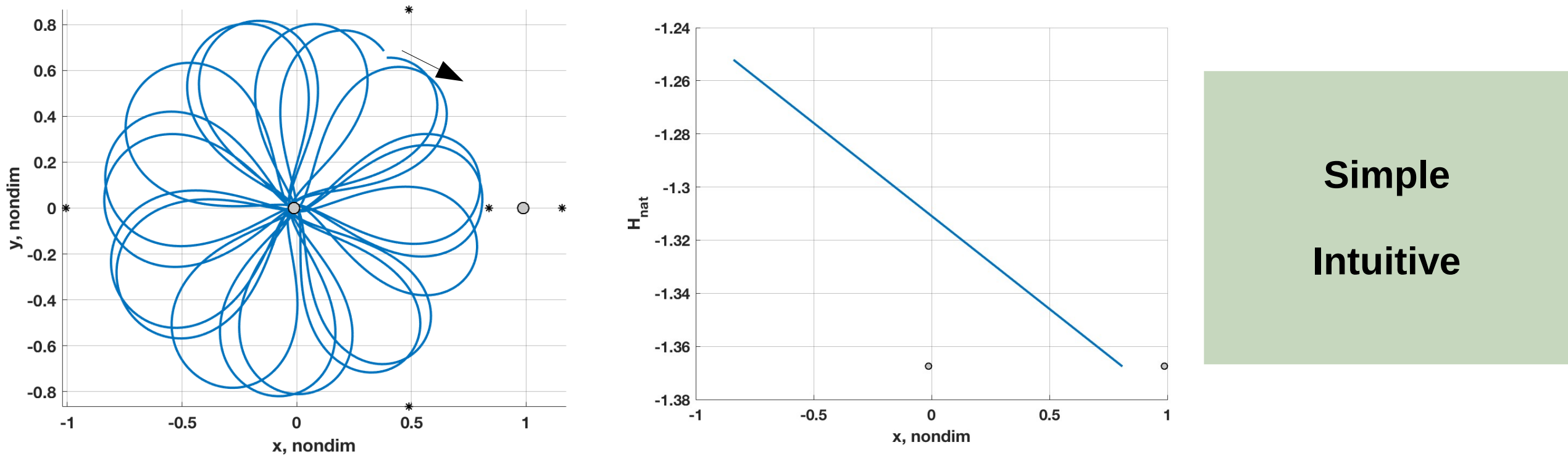

${ }_{275}$ Earth-Moon CR3BP-LT, $\alpha=180^{\circ}, a_{l t}=7 e-2$
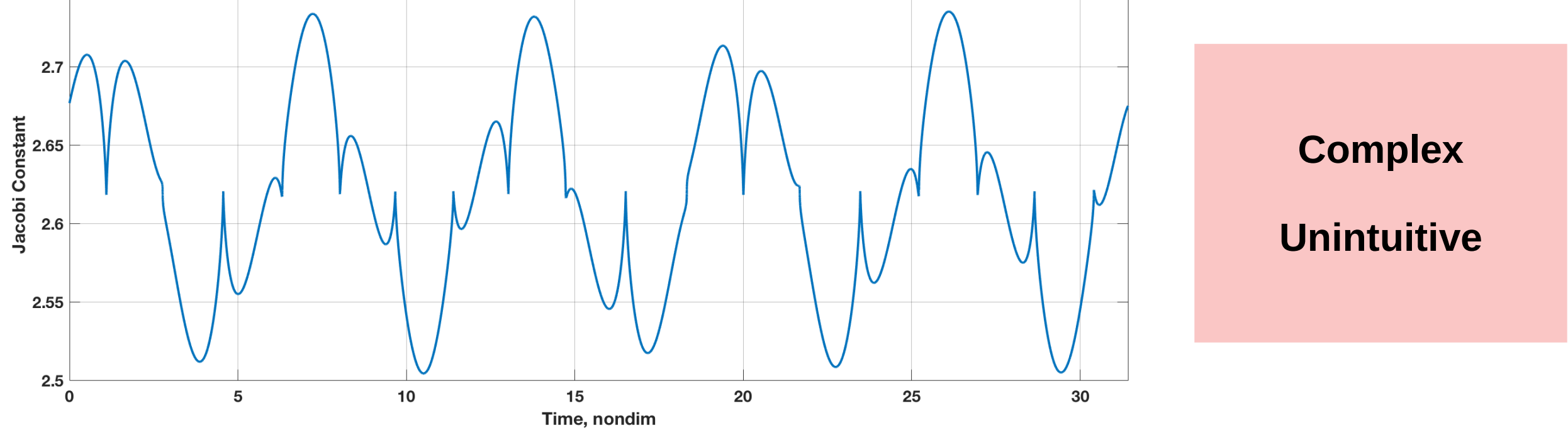

21 Aug 2018

Cox, Howell, Folta 


\section{Roadmap}

CR3BP

CR3BP-LT

Low-Thrust

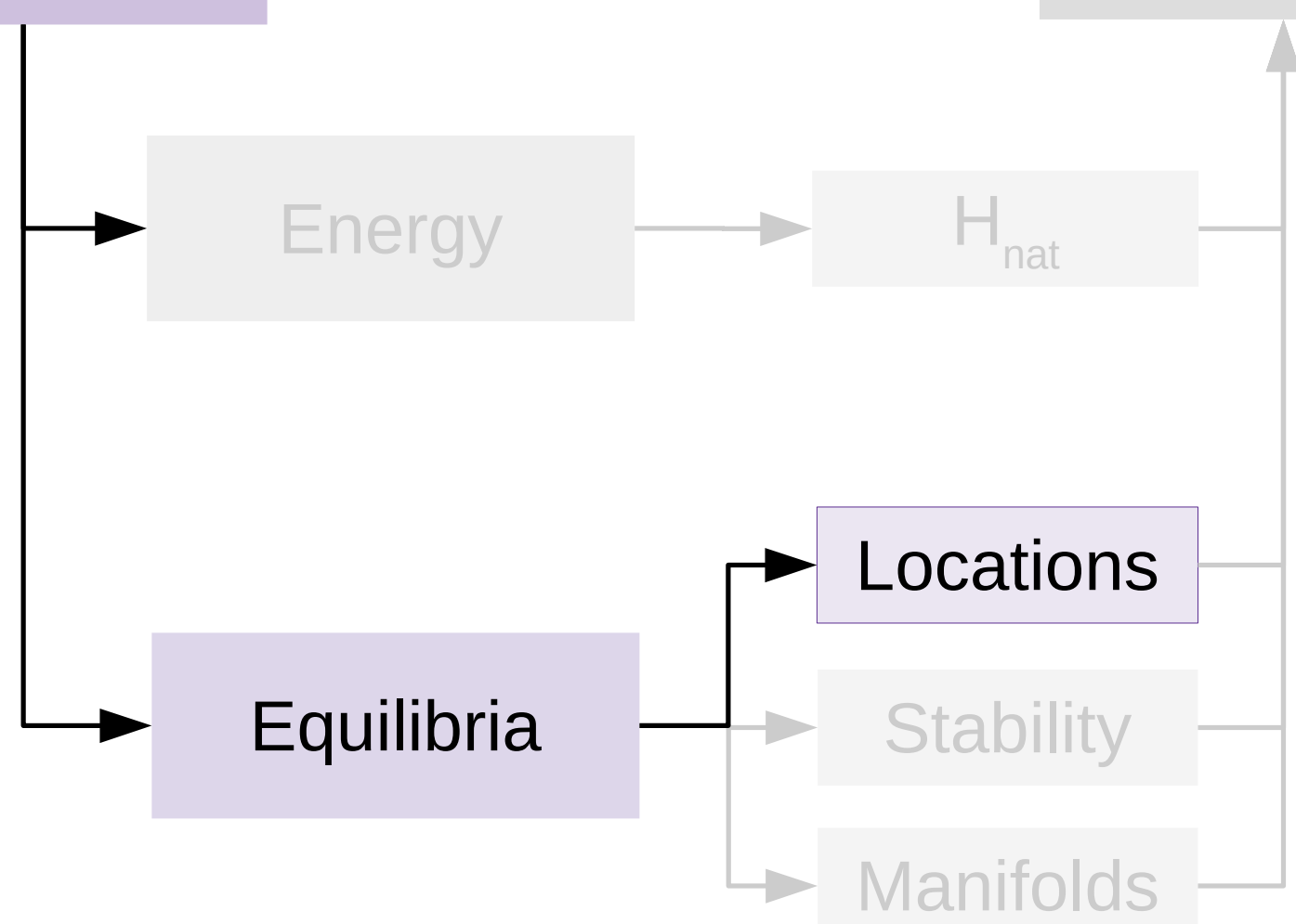




\section{Planar Low-Thrust Equilibria}

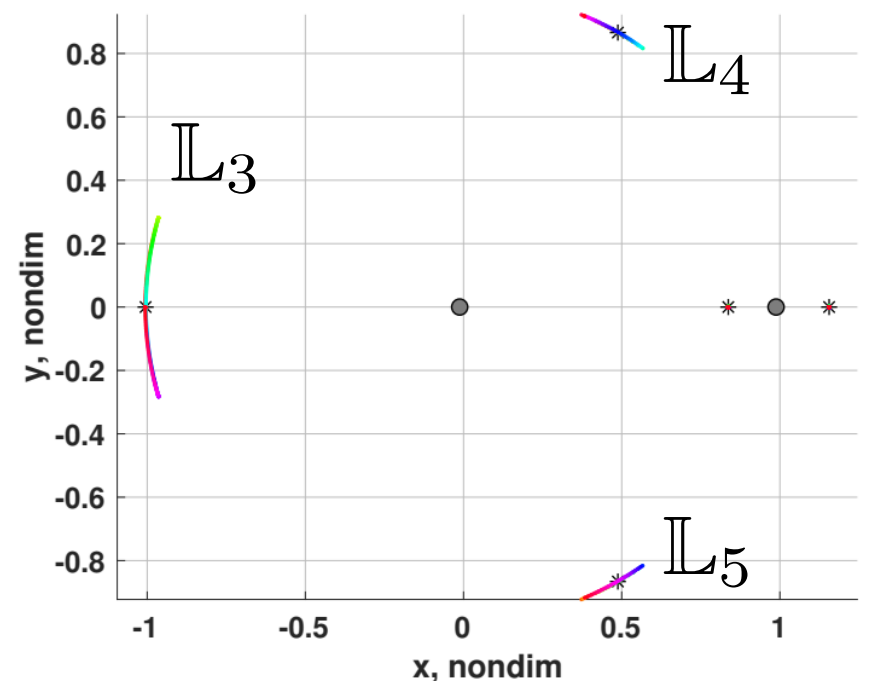

Small $\mathrm{a}_{\mathrm{lt}}$ value

$$
a_{l t}=8.73 e-3
$$

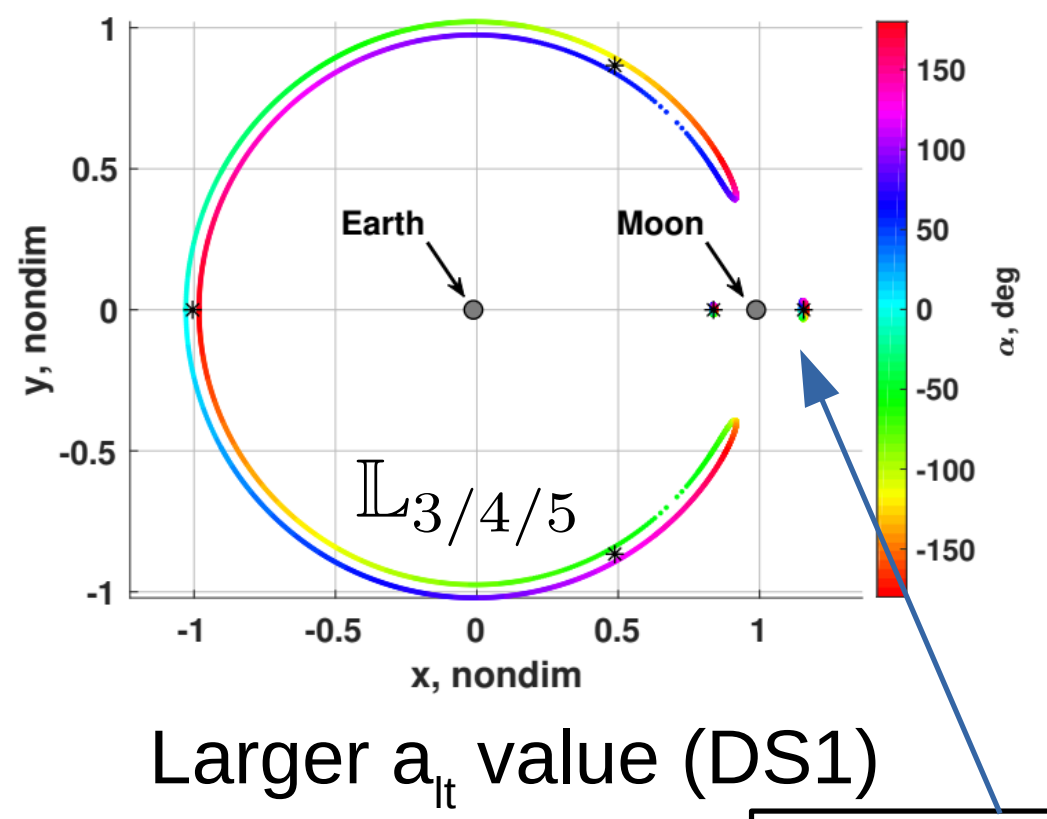

$$
a_{\mathrm{lt}}=7.0 \mathrm{e}-2
$$

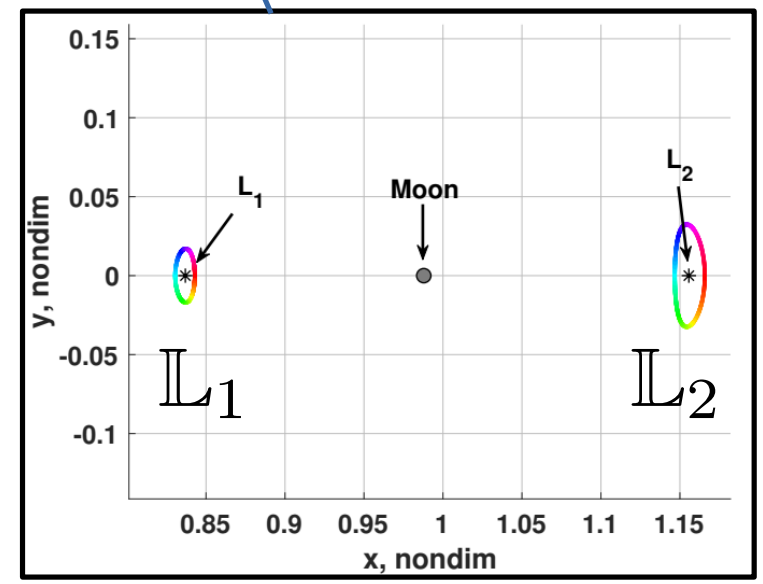

Cox, Howell, Folta

$12 / 38$ 


\section{Distinct Equilibrium Points}
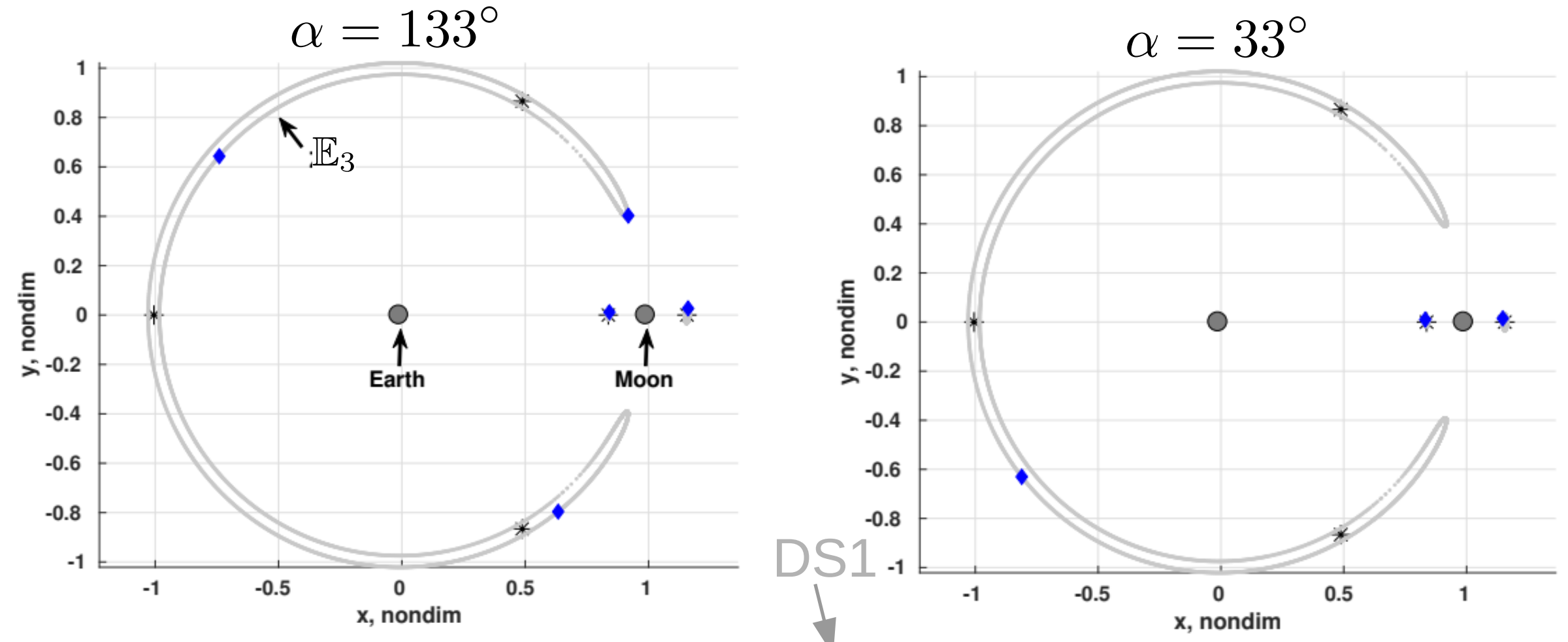

Earth-Moon CR3BP-LT, $a_{l t}=7.00 \mathrm{e}-2, \beta=0$

Accel. Mag. and direction determine equilibria locations and count 


\section{Roadmap}

CR3BP

CR3BP-LT

Low-Thrust

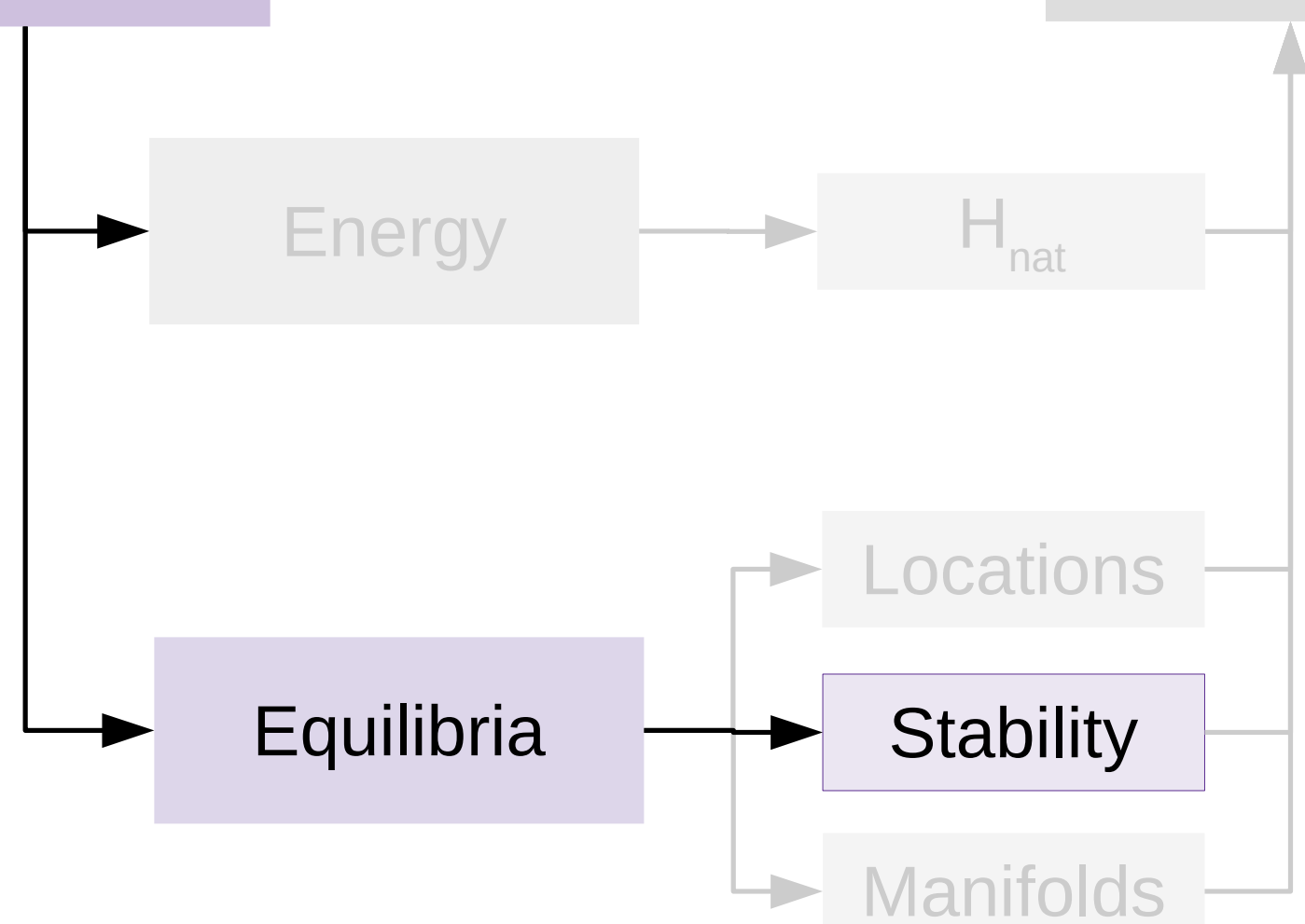




\section{Equilibria Stability}

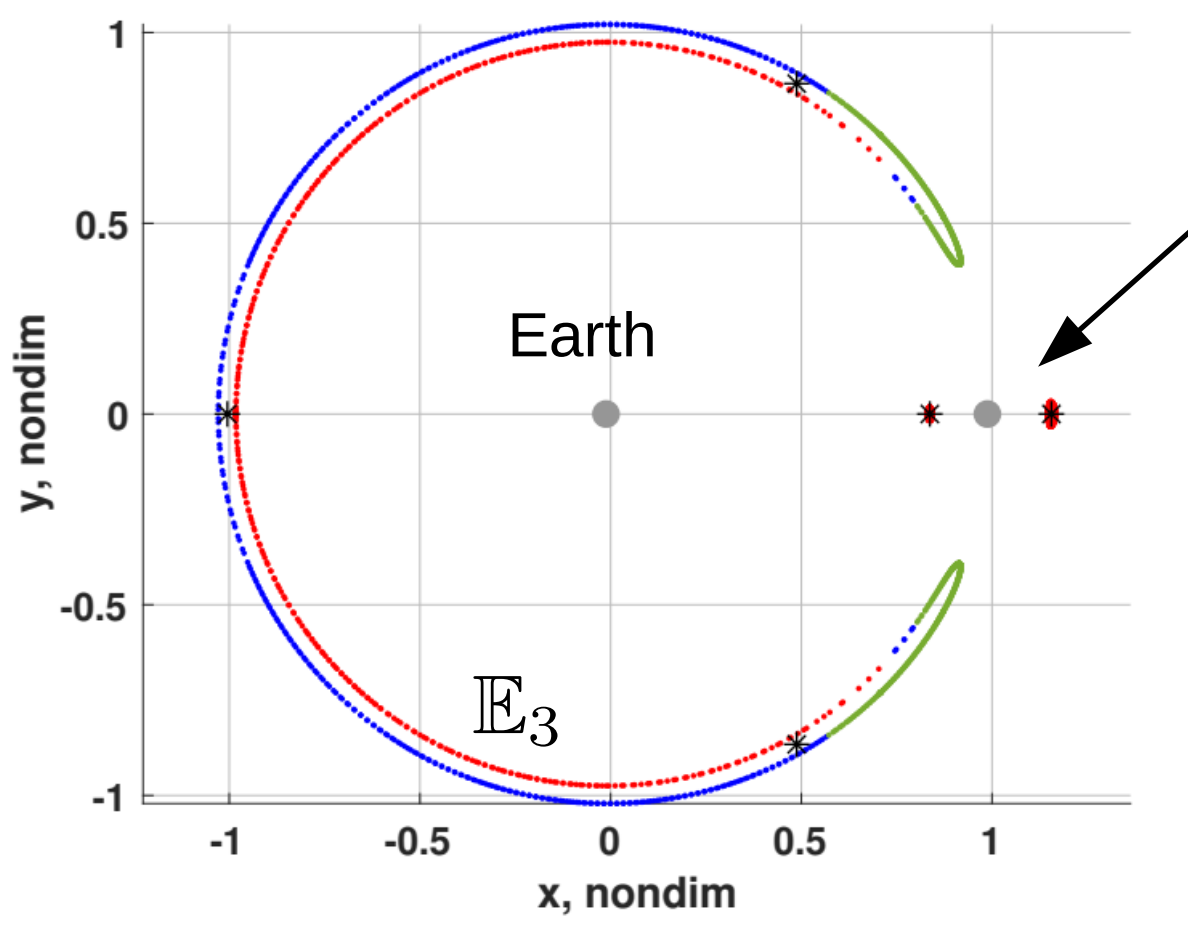

Natural CR3BP

- $\mathrm{L}_{1}, \mathrm{~L}_{2}, \mathrm{~L}_{3}-\mathrm{SxC}$

- $\mathrm{L}_{4}, \mathrm{~L}_{5}-\mathrm{CxC}$

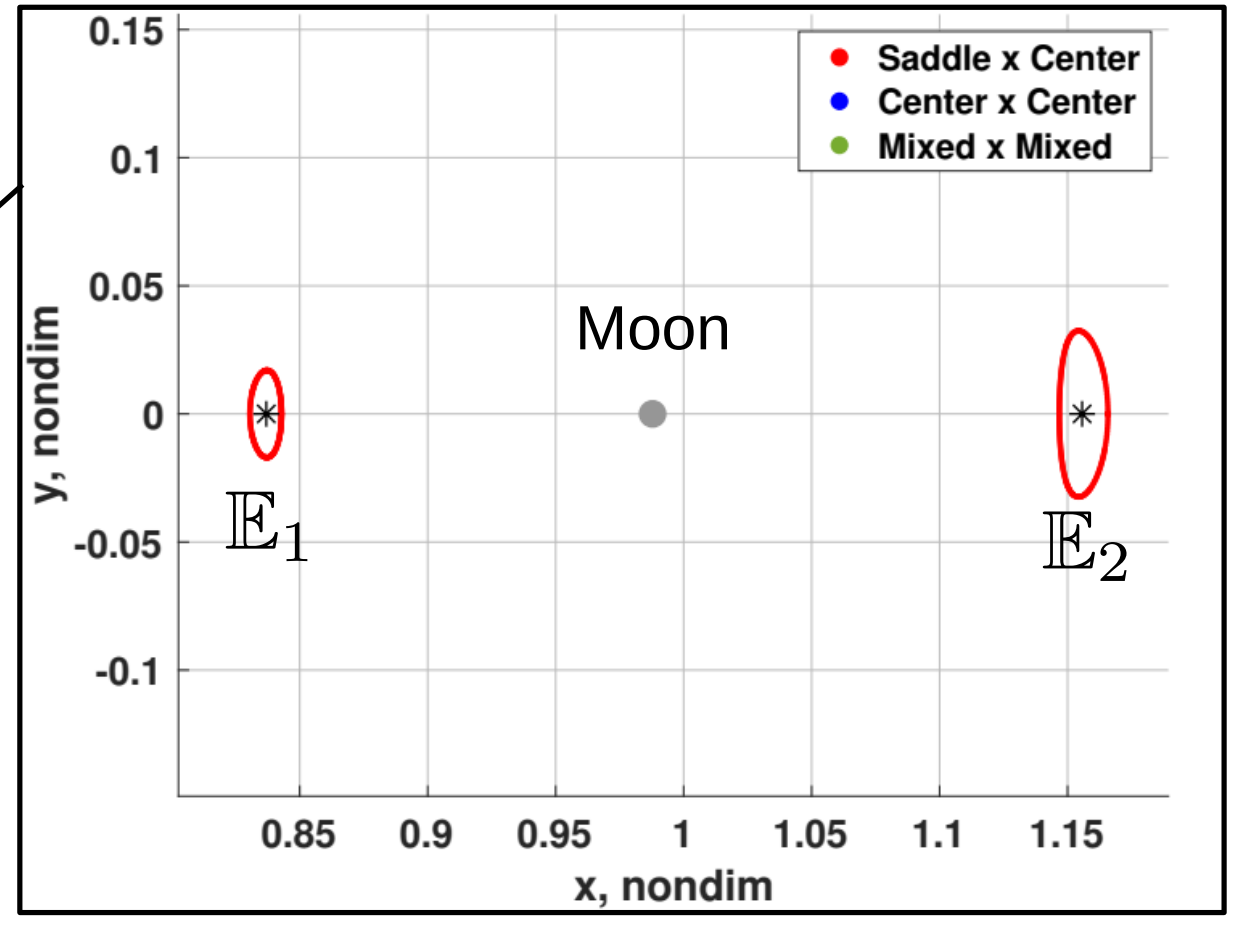

CR3BP-LT for $\mathrm{a}_{\mathrm{lt}}=7 \mathrm{e}-2$

- $\mathbb{E}_{1}, \mathbb{E}_{2}-$ SxC

- $\mathbb{E}_{3}-$ CxC, SxC, \& MxM 


\section{Near $L_{5}$}

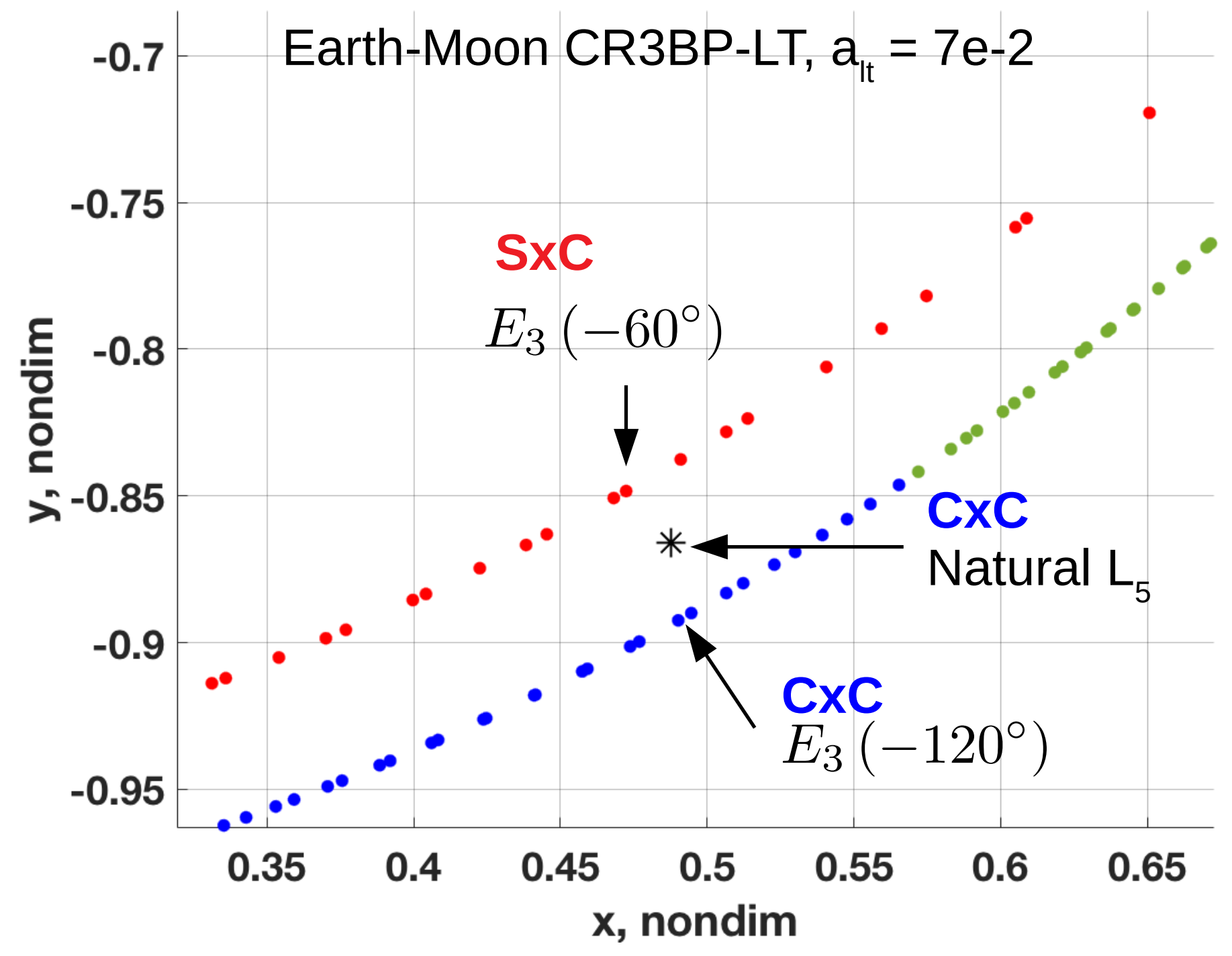




\section{Roadmap}

CR3BP

CR3BP-LT

Low-Thrust

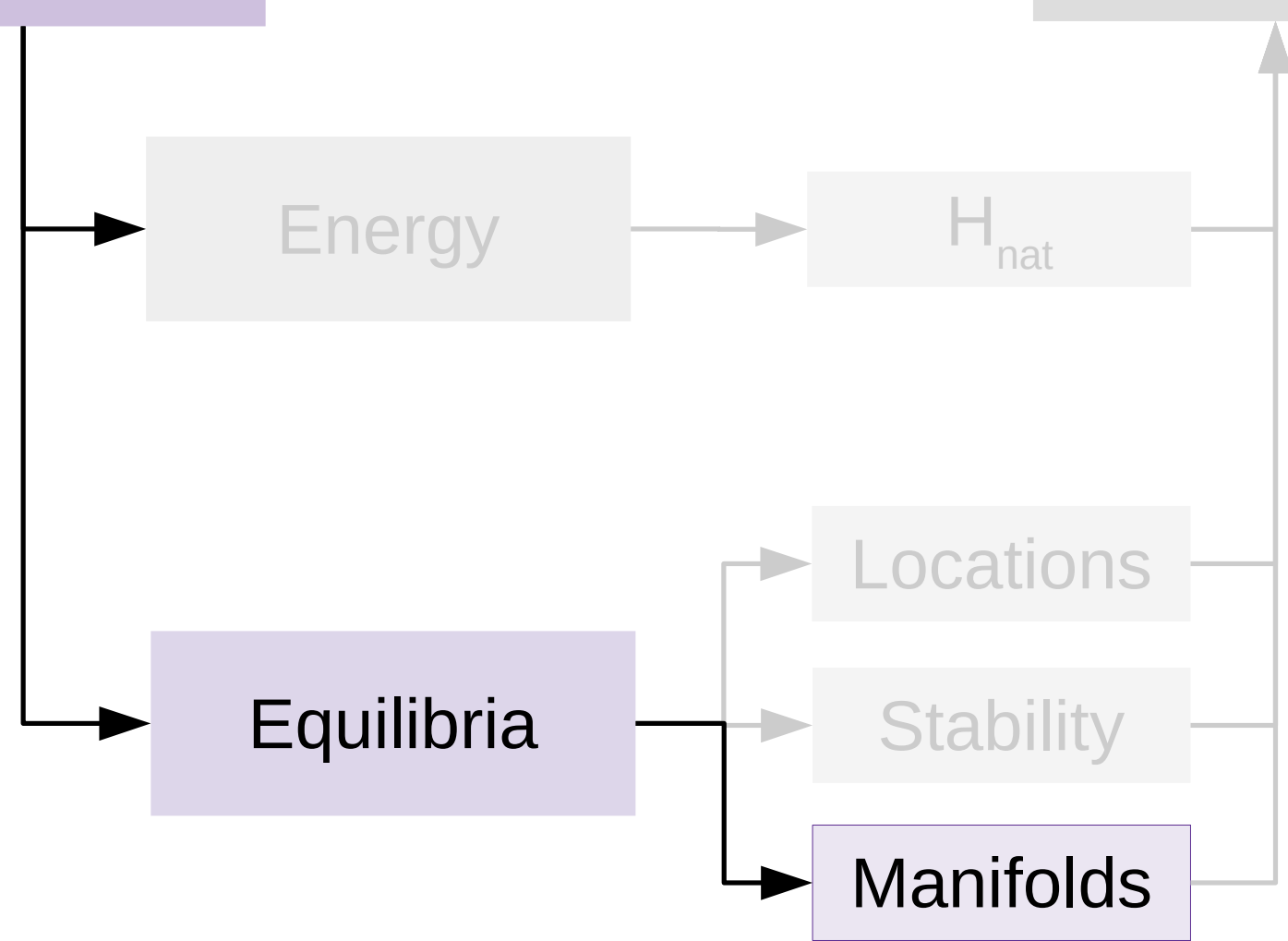




\section{Equilibria Manifolds}
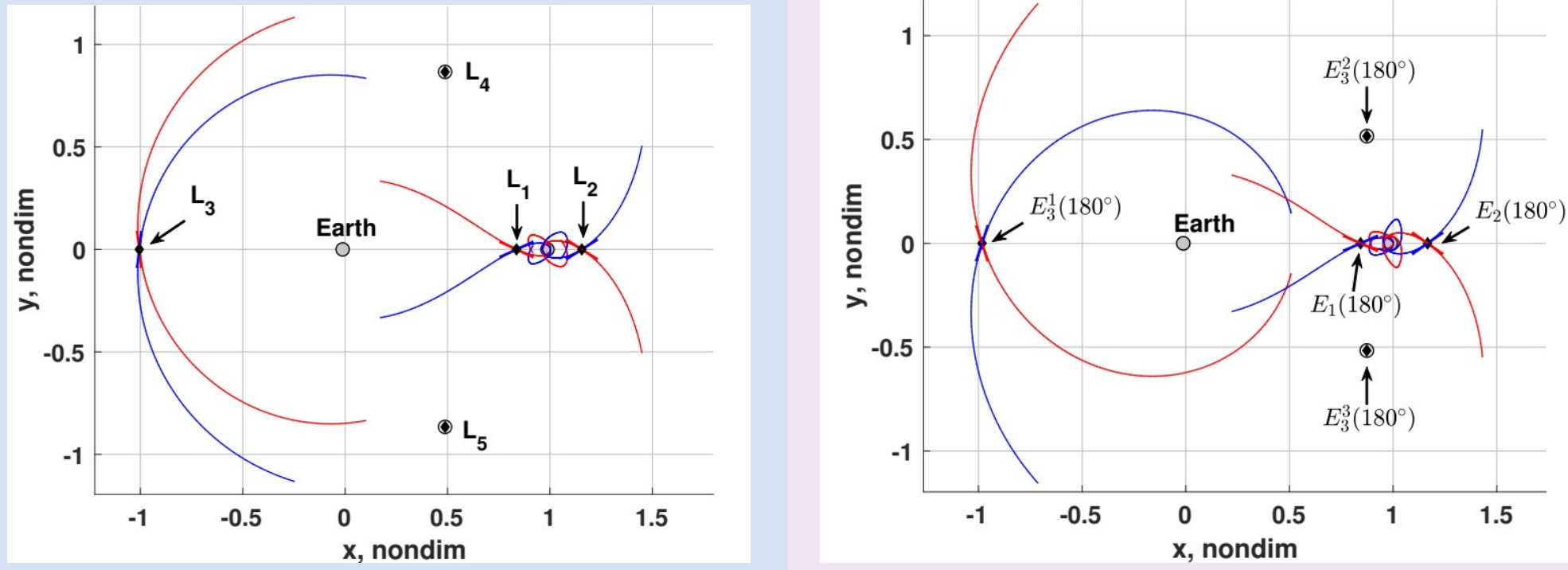

CR3BP-LT $\mathrm{a}_{\mathrm{tt}}=7 \mathrm{e}-2$
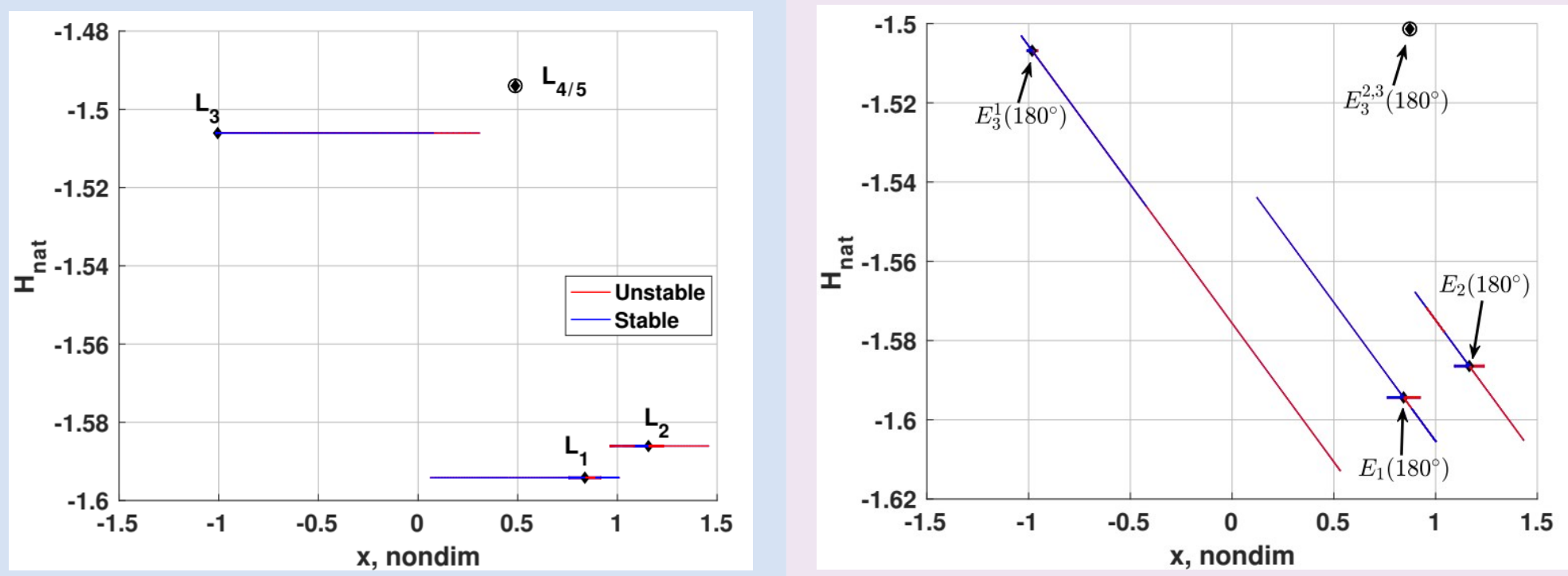
$\alpha=180^{\circ}$ 


\section{Equilibria Manifolds}

Earth-Moon CR3BP-LT $\mathrm{a}_{\mathrm{lt}}=7 \mathrm{e}-2$
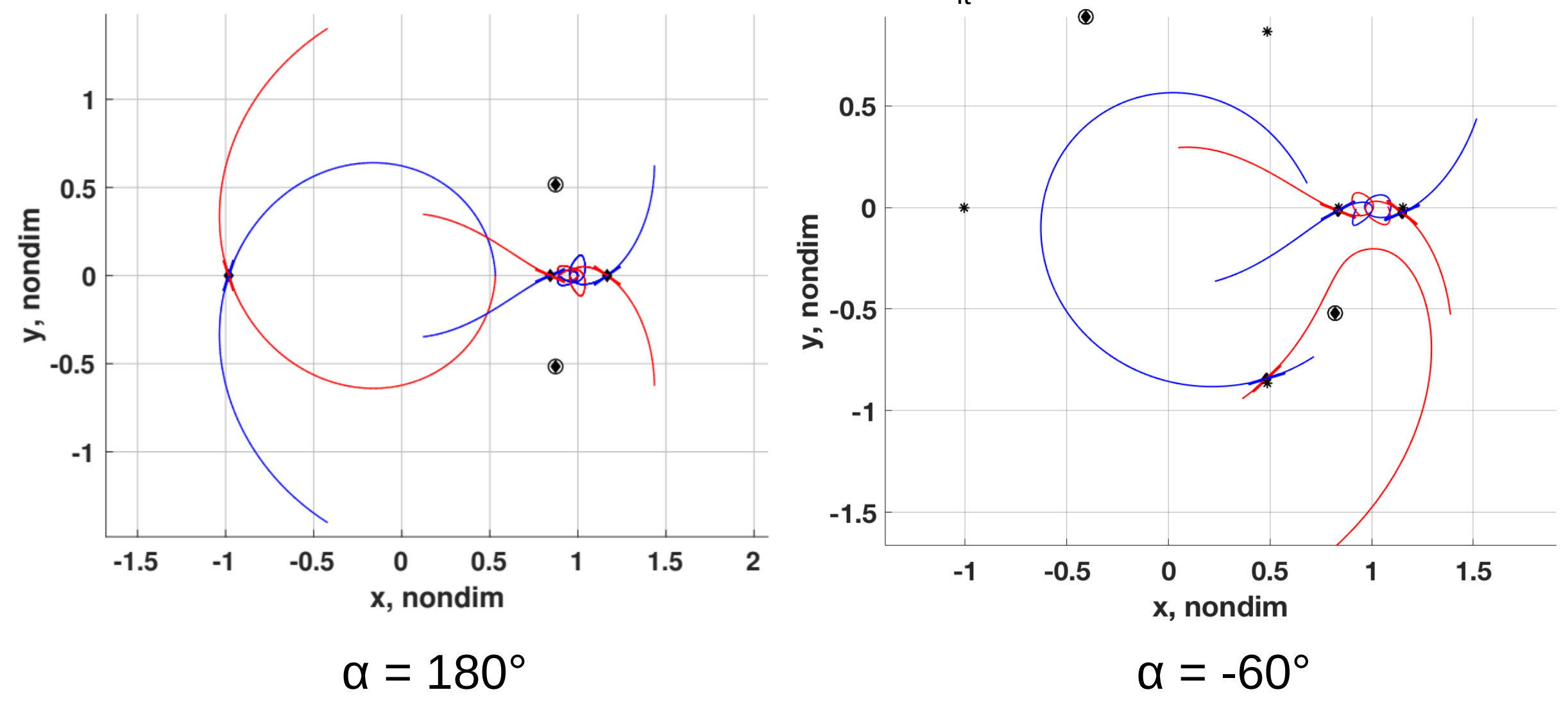


\section{Roadmap}

CR3BP

CR3BP-LT

Low-Thrust
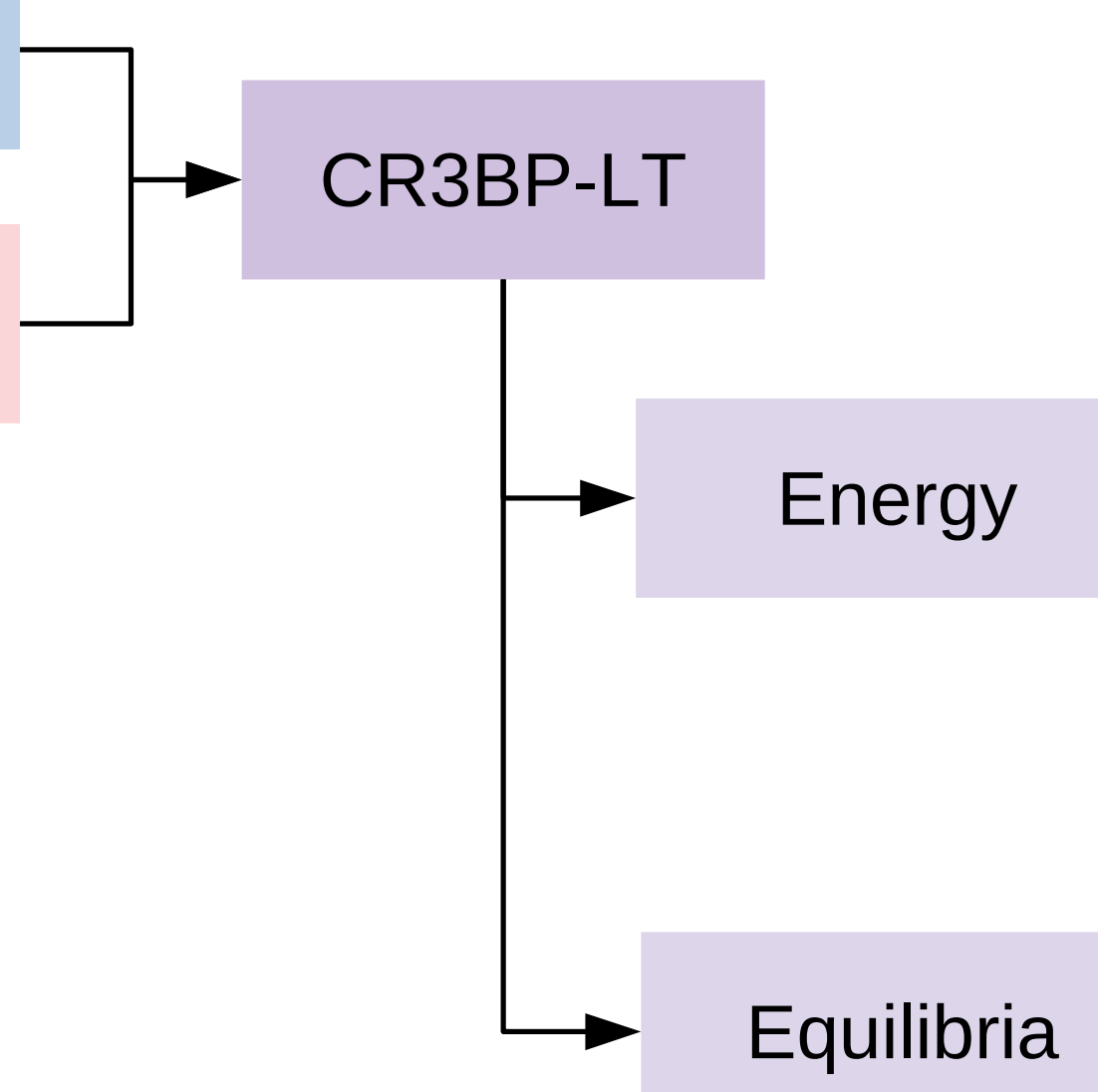

Design 


\section{Transit Design: Setup}

- Demonstrate use of dynamical structures and techniques

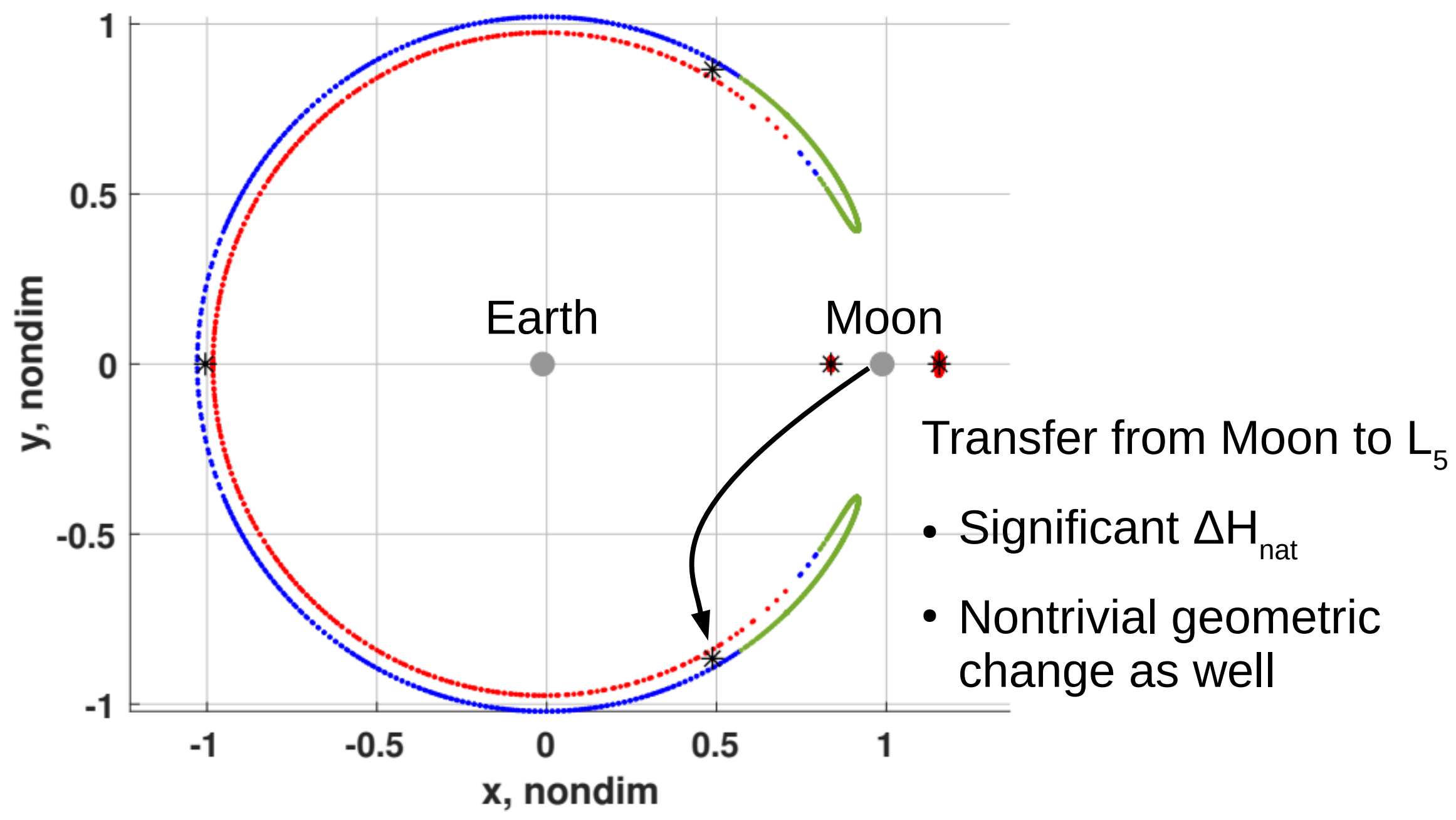




\section{Use Natural Structures?}

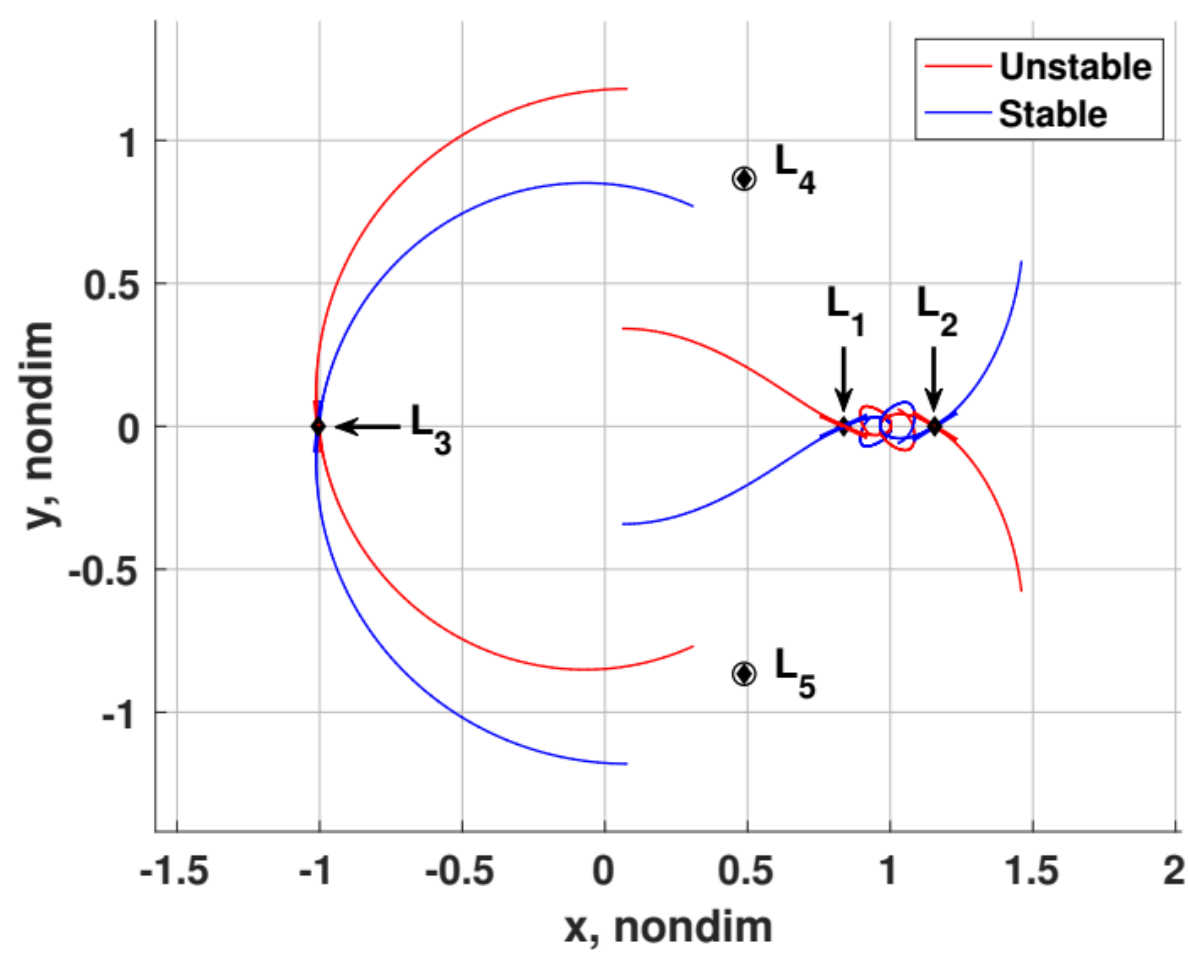

- $L_{1}$ and $L_{2}$ - saddle mode, offer access to moon

- $\mathrm{L}_{5}$ - no saddle mode

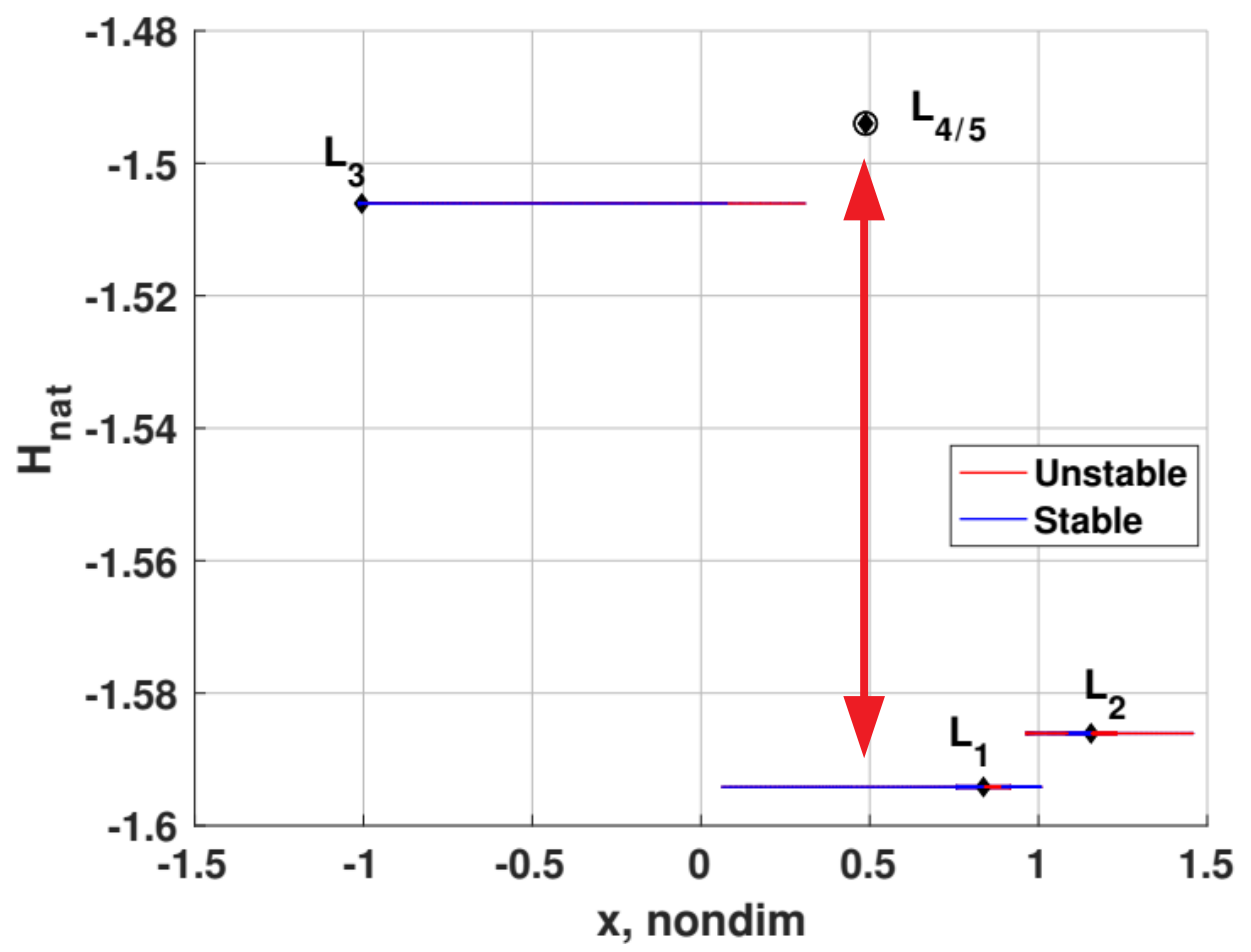

- Even with favorable geometry, energy difference is large

- Natural manifolds do not supply energy change 


\section{Use Low-Thrust Structures?}

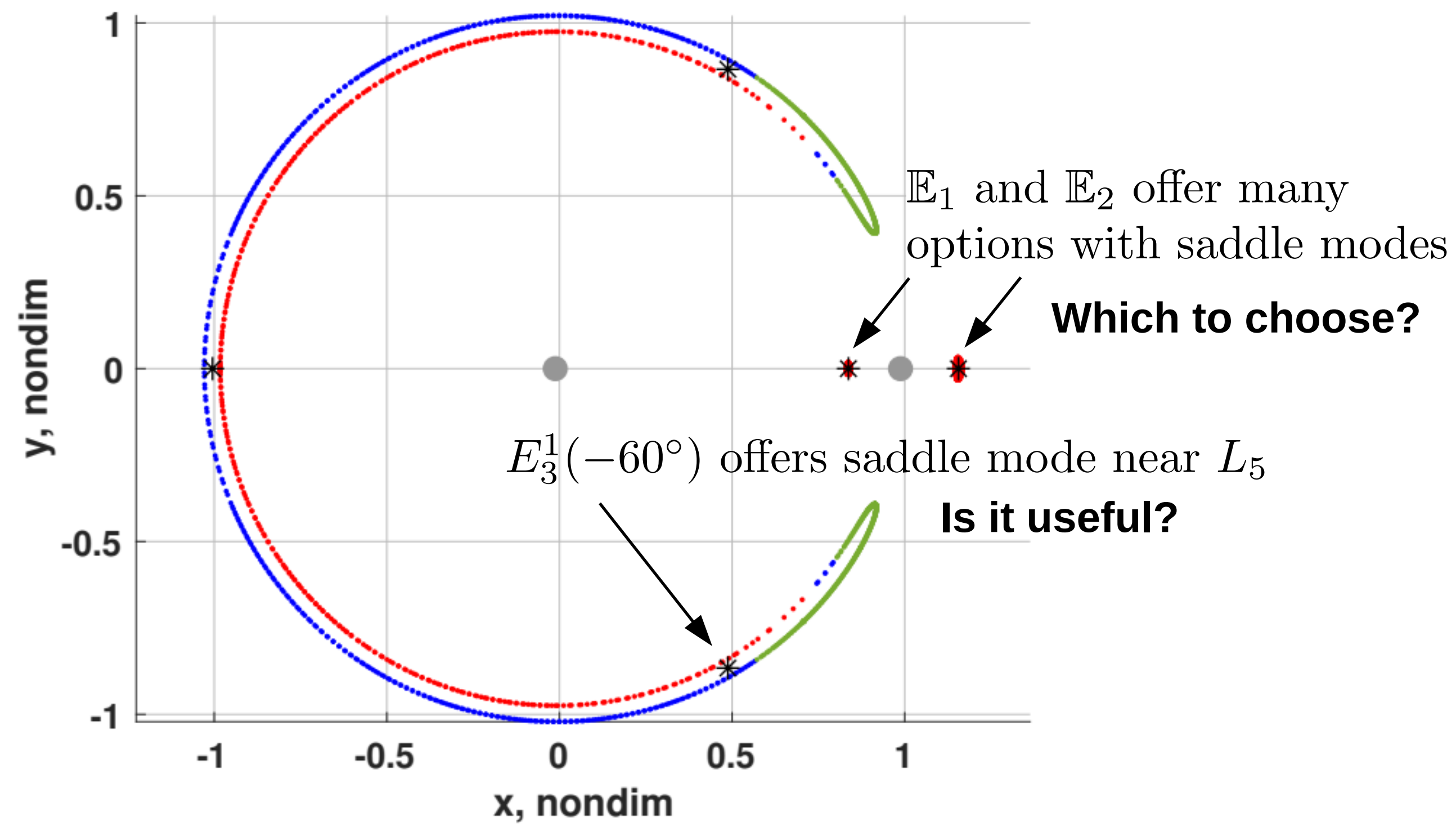




\section{Low-Thrust Structures}

1. Choose $\mathbb{E}_{2}$ (arbitrary)

2. Use energy plane to select equilibrium point with maximum $\mathrm{H}_{\text {nat }}$ increase
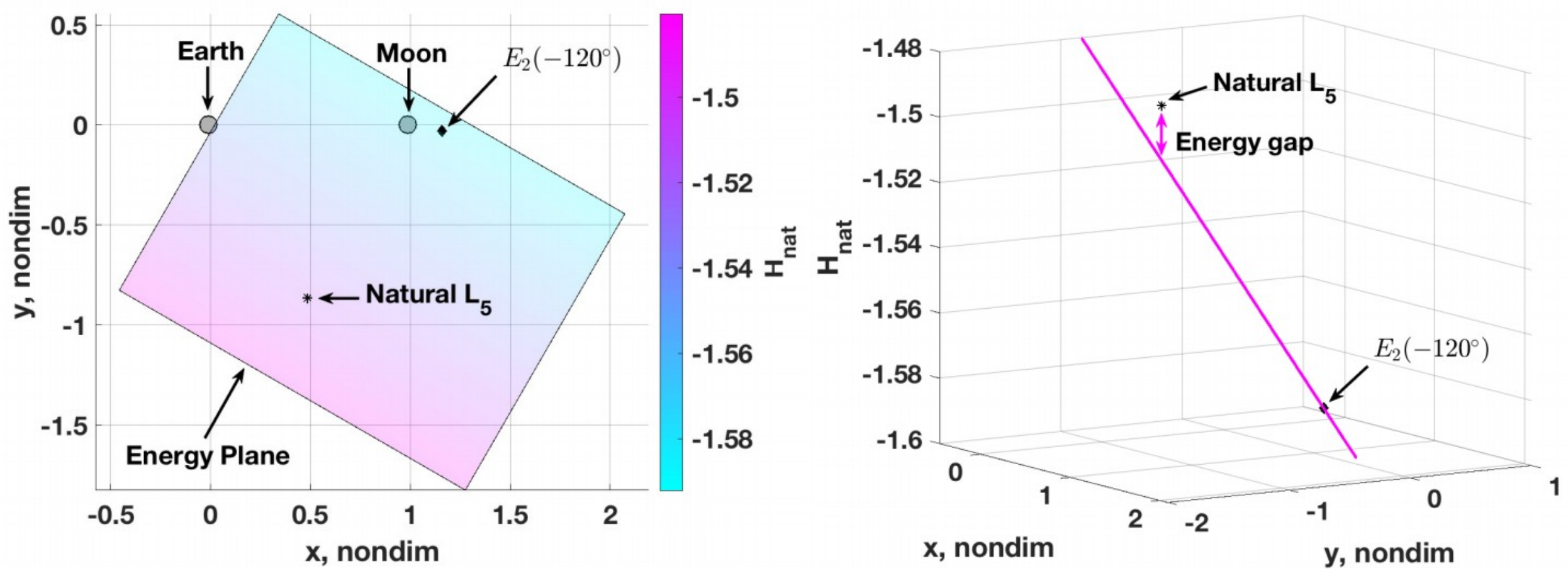


\section{Additional Structures}

3. Include $E_{3}^{1}\left(-60^{\circ}\right)$ stable manifolds (blue)

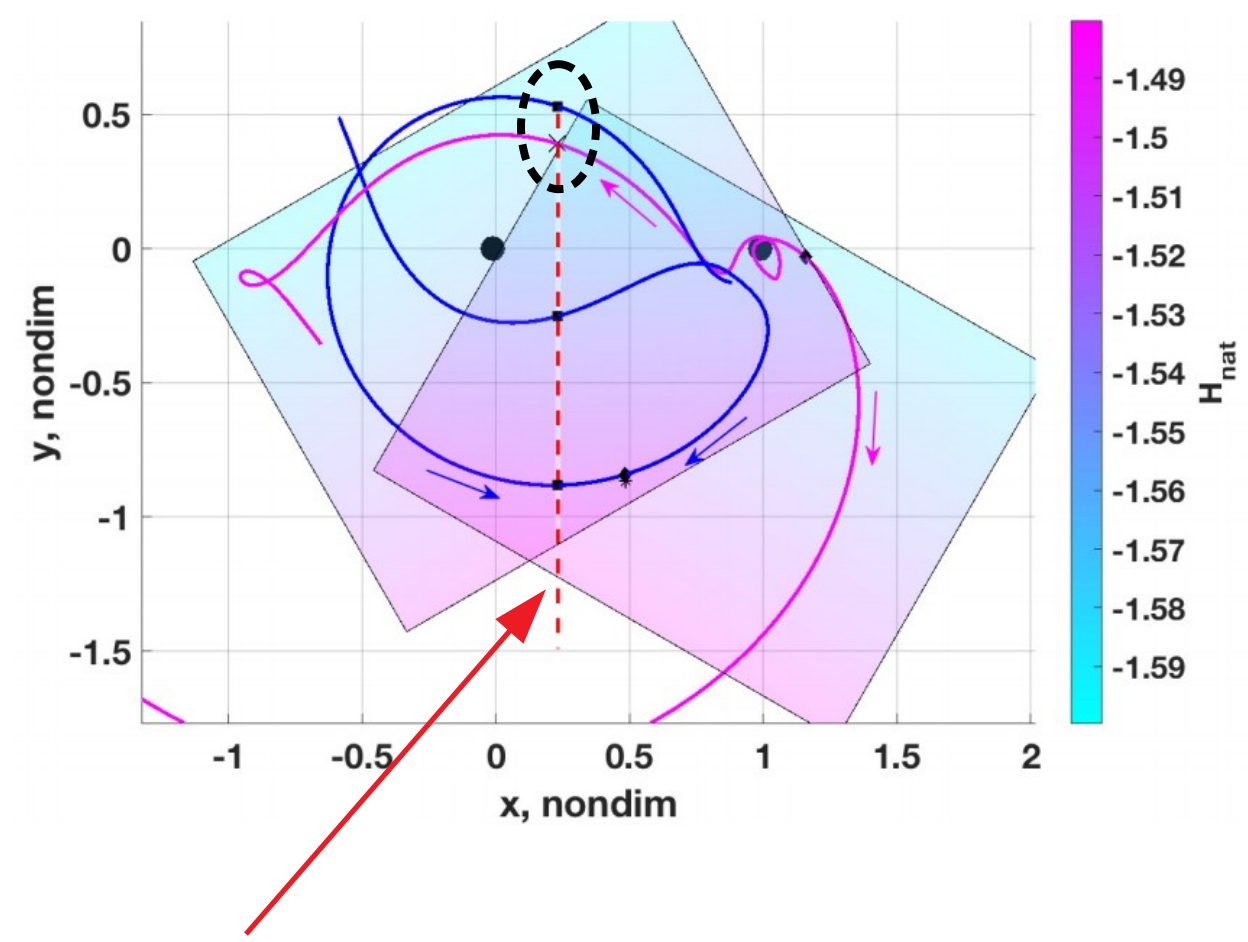

4. Select candidate arcs that nearly intersect in $x, y$, and $H_{\text {nat }}$

Intersection of two energy planes is convenient hyperplane: nearby points possess similar positions and energies 


\section{Sample Transfer: Initial Design}
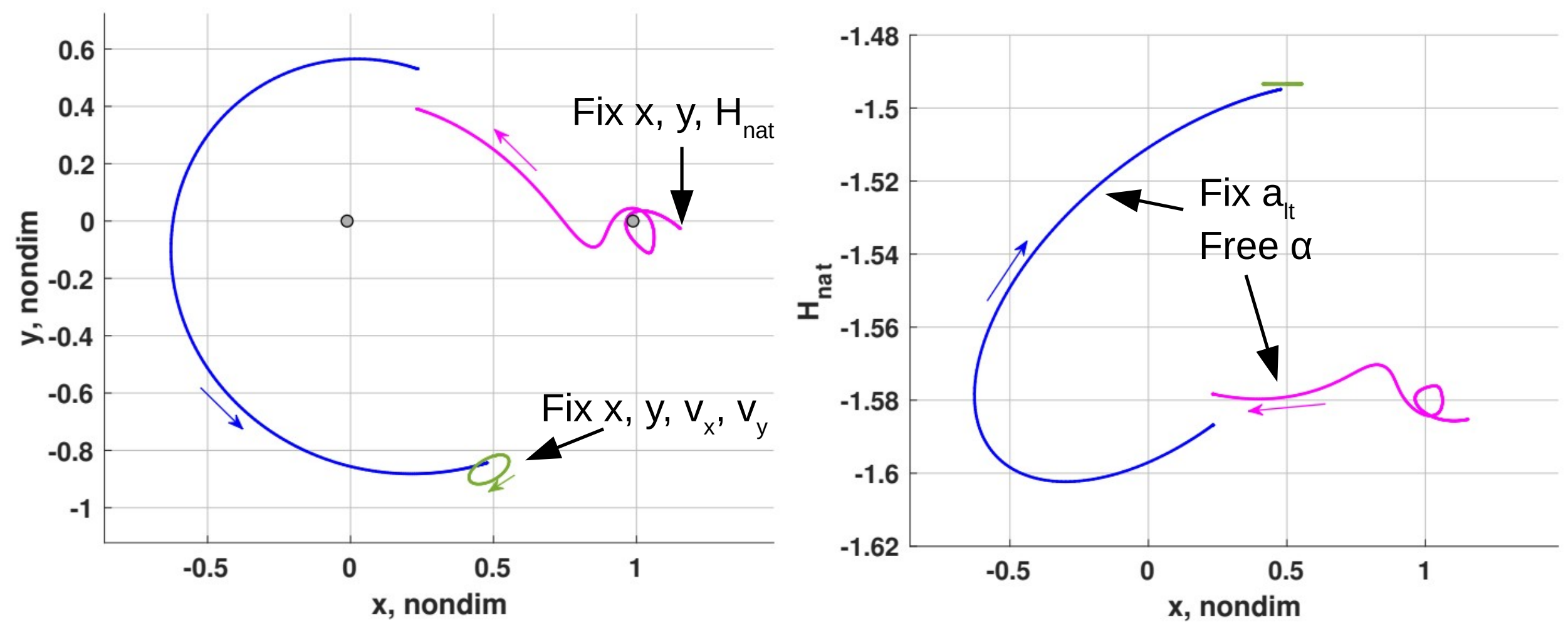

5. Include $L_{5}$ short period orbit (SPO) near destination to maintain proximity to $L_{5}$

6. Discretize into smaller arcs

7. Correct for continuity 


\section{Sample Transfer: Feasible Sol'n}
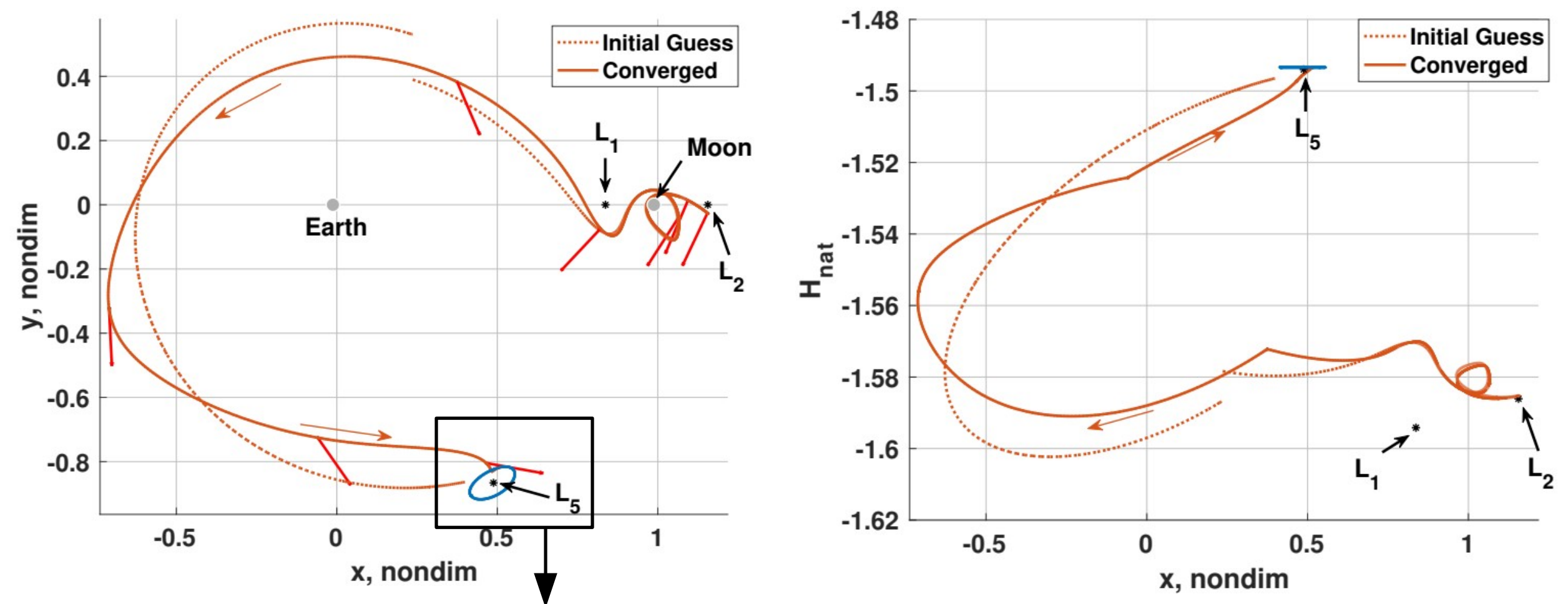

- Rapid convergence

- Initial geometry and energy profile preserved (unsurprising; min-norm) 


\section{Conclusions}

- Reasonable assumptions yield conservative, autonomous CR3BP-LT

- Low-thrust equilibria possess diverse locations \& stability, function of thrust vector magnitude and orientation

- Energy along low-thrust arc confined to a plane oriented by thrust vector properties

- Links between thrust vector and arc geometry \& energy facilitate initial design for thrust vector 


\section{Acknowledgements}

Purdue Multi-Body Dynamics Research Group

Purdue University School of Aeronautics and Astronautics

JPL Mission Design and Navigation Branch

Dan Grebow

This work was supported by a NASA Space Technology Research

Fellowship, grant number NNX16AM40H 


\section{Trajectory Design Leveraging Low-thrust, Multi-Body \\ Equilibria and Their Manifolds}

Andrew D. Cox | Kathleen C. Howell | David C. Folta

August 21, 2018

AAS/AIAA Astrodynamics Specialist Conference

Snowbird, UT 


\section{Backup Slides}




\section{Thrust Magnitude}

\begin{tabular}{lcccccc} 
& & & $f=\frac{F t_{*}^{2}}{1000 l_{*} M_{3,0}}$ \\
\hline Spacecraft & $M_{3,0}$ & $F$ & $a_{l t}$ & $f$ (Earth-Moon) & $f$ (Sun-Earth) \\
& $\mathrm{kg}$ & $\mathrm{mN}$ & $\mathrm{m} / \mathrm{s}^{2}$ & nondim & $3.19 \mathrm{e}-2$ \\
& 486 & 92.0 & $1.893 \mathrm{e}-4$ & $6.95 \mathrm{e}-2$ & $0.79 \mathrm{e}-2$ \\
\hline Deep Space $1^{1}$ & 510 & 24.0 & $4.706 \mathrm{e}-5$ & $1.73 \mathrm{e}-2$ & $1.28 \mathrm{e}-2$ \\
Hayabusa $^{2}$ & 1218 & 92.7 & $7.611 \mathrm{e}-5$ & $2.79 \mathrm{e}-2$ & $1.20 \mathrm{e}-2$ \\
Dawn $^{3}$ & 14 & 1.0 & $7.143 \mathrm{e}-5$ & $2.62 \mathrm{e}-2$ & \\
Lunar IceCube $^{4}$ & & & & & \\
\hline
\end{tabular}

Nondimensional magnitude between $1 \mathrm{e}-2$ and $1 \mathrm{e}-1$ is "reasonable" 


\section{Hamiltonian Time Derivative(s)}

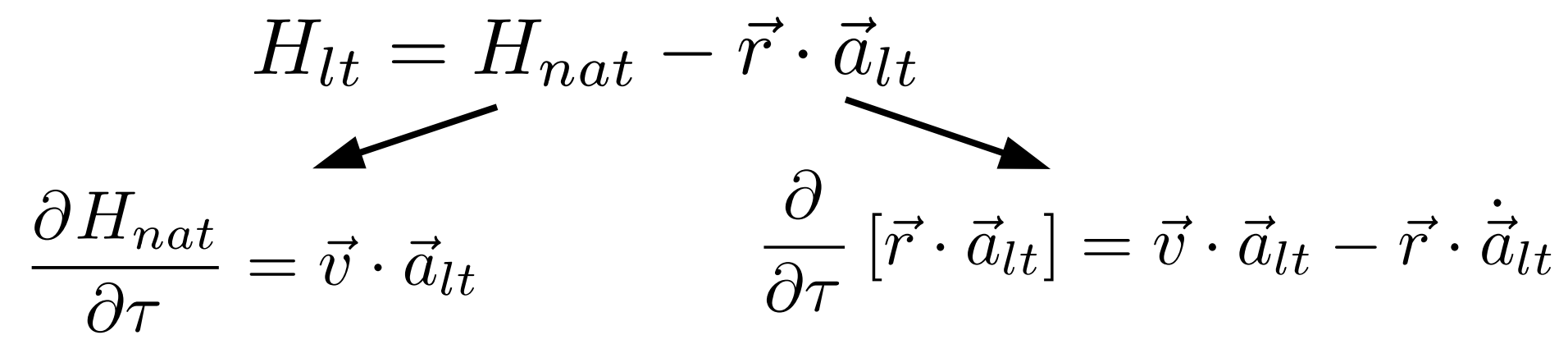

$\hat{u} \perp \vec{v} \quad \mathrm{H}_{\text {nat }}=$ const.

$\hat{u} \| \vec{v} \quad \begin{aligned} & \text { Maximize } \mathrm{H}_{\text {nat }} \\ & \text { rate of change }\end{aligned}$
If $\quad \dot{\vec{a}}_{l t}=\overrightarrow{0}$

then $\frac{\partial}{\partial \tau}\left[\vec{r} \cdot \vec{a}_{l t}\right]=\vec{v} \cdot \vec{a}_{l t}$

and $\mathrm{H}_{\mathrm{It}}=$ const. $^{*}$

*Demonstrated reasonable via Monte Carlo with variable $\mathrm{a}_{\mathrm{lt}}$ in Earth-Moon system 


\section{Energy-Like Quantities}

CR3BP-LT system:

$$
H_{l t}=H_{n a t}-\vec{r} \cdot \vec{a}_{l t}
$$

- Constant on low-thrust arcs when $\mathbf{a}_{\mathrm{lt}}$ is const. (mag., angle)

- Rapidly changed by varying $\mathrm{a}_{\mathrm{lt}}$ or $\alpha$ 


\section{Energy Plane Proof}

$$
\begin{aligned}
\Delta \vec{\rho}= & \vec{\rho}\left(\tau_{2}\right)-\vec{\rho}\left(\tau_{1}\right)=\left(x_{2}-x_{1}\right) \hat{x}+\left(y_{2}-y_{1}\right) \hat{y}+\left(H_{n a t, 2}-H_{n a t, 1}\right) \hat{H} \\
= & \Delta x \hat{x}+\Delta y \hat{y}+\Delta H \hat{H} \\
= & {\left[\Delta x C_{\alpha} C_{\gamma}+\Delta y S_{\alpha} C_{\gamma}-\Delta H S_{\gamma}\right] \hat{x}^{\prime \prime}+\left[\Delta y C_{\alpha}-\Delta x S_{\alpha}\right] \hat{y}^{\prime \prime}+} \\
& \quad\left[\Delta x C_{\alpha} S_{\gamma}+\Delta y S_{\alpha} S_{\gamma}+\Delta H C_{\gamma}\right] \hat{H}^{\prime \prime}
\end{aligned}
$$

For in-plane motion: $\Delta \vec{\rho} \cdot \hat{H}^{\prime \prime}=0$

$$
\begin{aligned}
\Delta \vec{\rho} \cdot \hat{H}^{\prime \prime}=0 & =\Delta x C_{\alpha} S_{\gamma}+\Delta y S_{\alpha} S_{\gamma}+\Delta H C_{\gamma} \\
& =\Delta H+\tan \gamma\left[\Delta x C_{\alpha}+\Delta y S_{\alpha}\right] \\
& =\Delta H-\vec{r} \cdot \vec{a}_{l t}
\end{aligned}
$$

Substitute $H_{\text {nat }}$ path invariance to yield zero on LHS 


\section{Low-Thrust Equilibria: Balance}

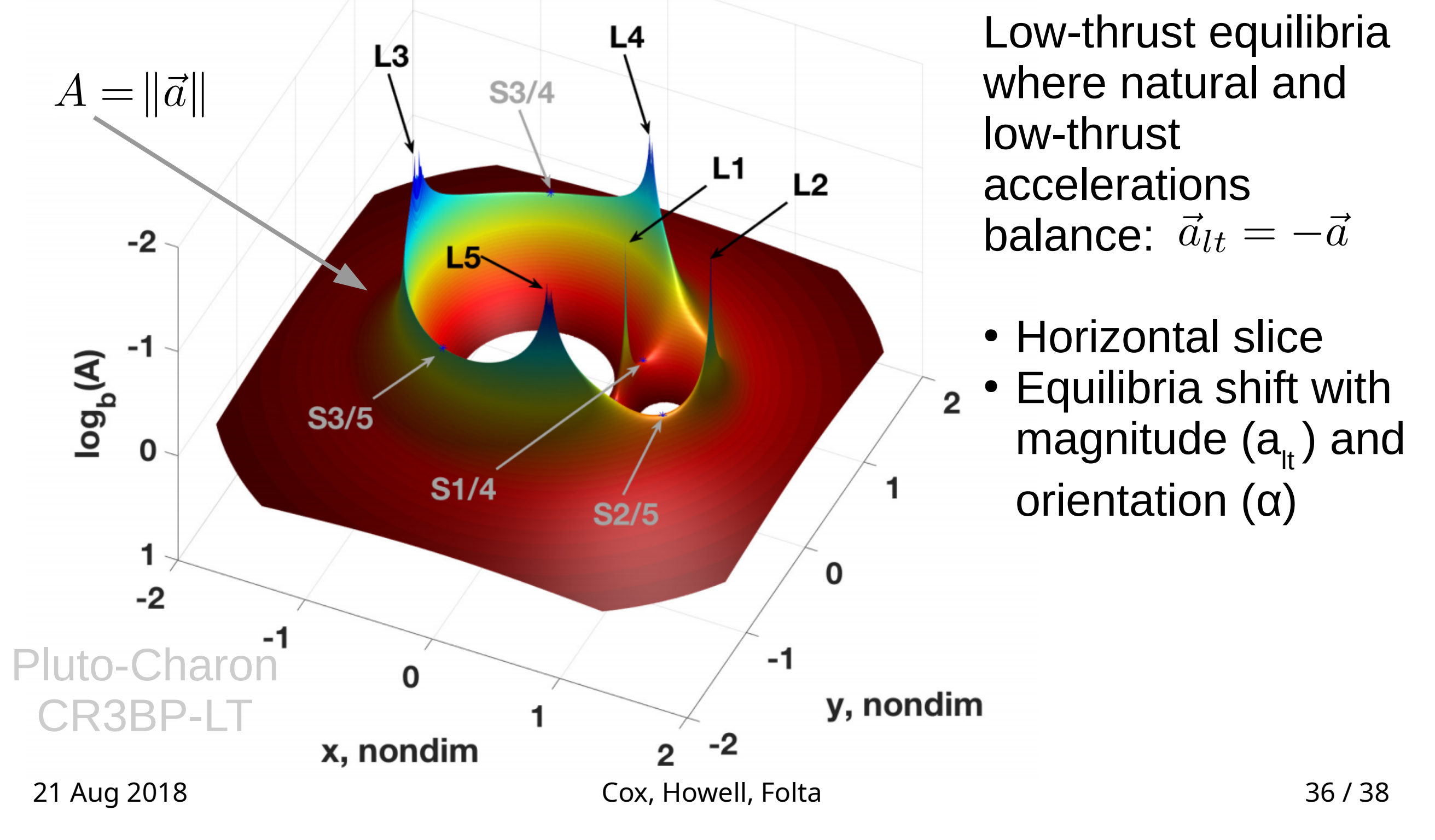




\section{Equilibria, Cont'd}
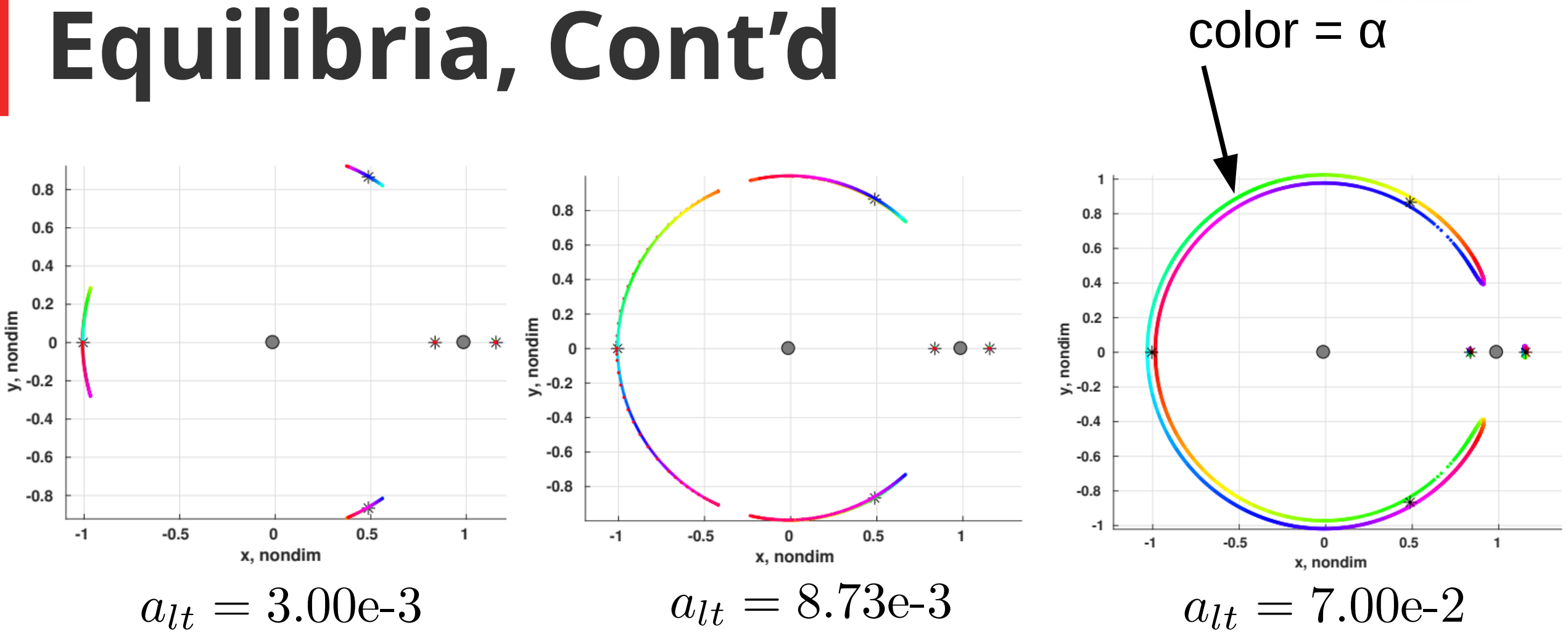

- At small $\mathrm{a}_{\mathrm{lt}}$ values: equilibria remain near CR3BP L pts (asterisks)

- Larger $\mathrm{a}_{\mathrm{lt}}$ values: equilibria form larger contours - "Zero Acceleration Contours" (ZACs) balance accelerations 


\section{Distinct Equilibria}

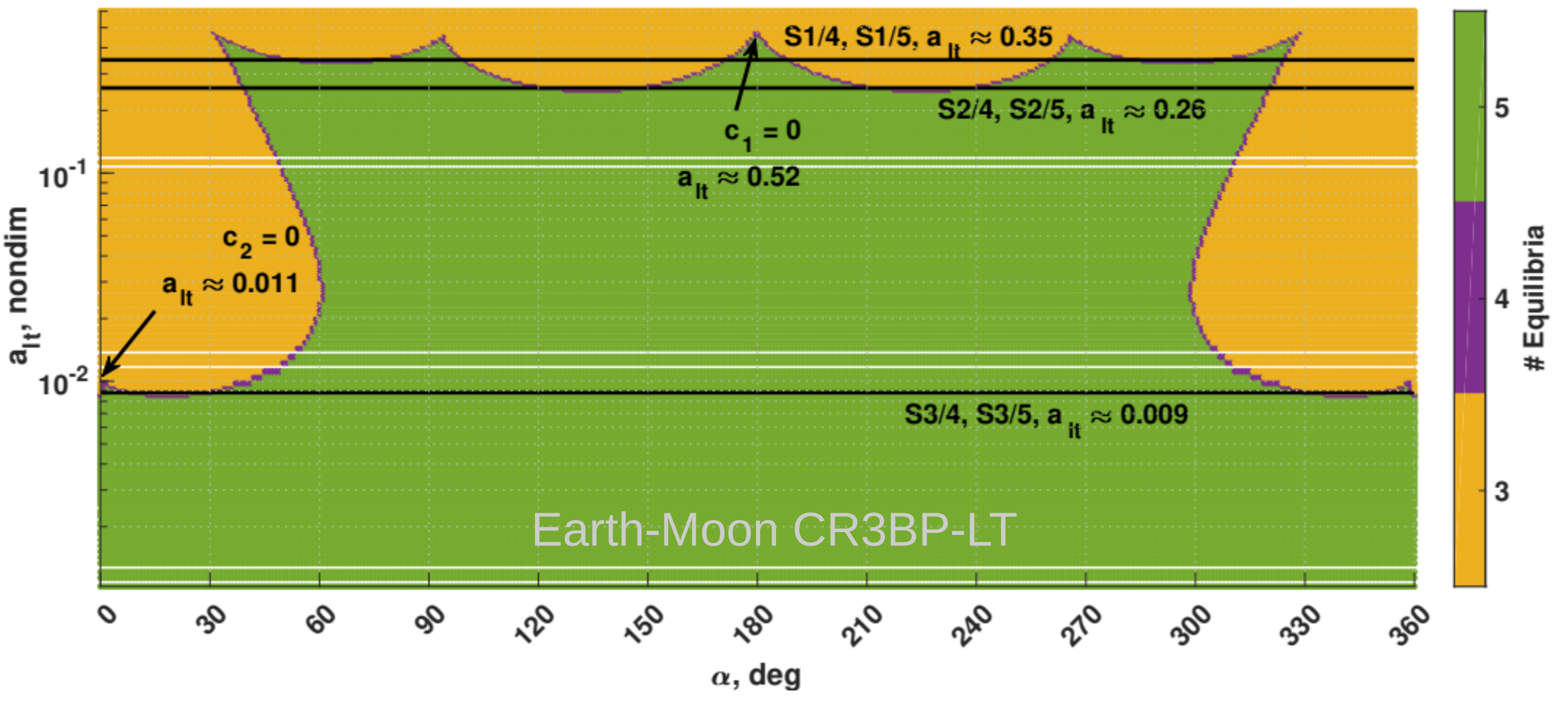

Number of distinct solutions varies with $\mathrm{a}_{\mathrm{lt}}$ and $\alpha$ 